\title{
Total Synthesis and Structure Revision of Diplobifuranylone B
}

Xinpeng Cheng, Carlos D. Quintanilla, and Liming Zhang*

Department of Chemistry \& Biochemistry, University of California, Santa Barbara, CA 93106

\section{E-mail: zhang@chem.ucsb.edu}

\begin{tabular}{|l|l|}
\hline \multicolumn{1}{|c|}{ Content } & Page number \\
\hline${ }^{1} \mathrm{H}$ and ${ }^{13}$ C NMR Comparison & $\mathrm{S} 2$ \\
\hline Chemical Shifts of the C3 of $\boldsymbol{\gamma}$-Lactones & $\mathrm{S} 4$ \\
\hline Comments about Literature Misassignment & $\mathrm{S} 5$ \\
\hline DFT Calculations & $\mathrm{S} 6$ \\
\hline References & $\mathrm{S} 9$ \\
\hline${ }^{1} \mathrm{H},{ }^{13} \mathbf{C},{ }^{1} \mathrm{H}$ Homonuclear Decoupling and $2 \mathrm{D}$ NMR spectra & $\mathrm{S} 10$ \\
\hline
\end{tabular}




\section{${ }^{1}$ H NMR Comparison}

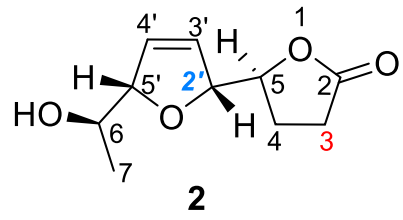

(6R, 5'S, 2'R, 5S)

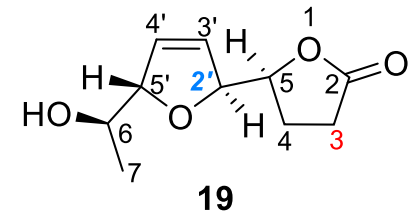

(6R, 5'S, 2'S, 5S)

\begin{tabular}{clll}
\hline Position & \multicolumn{1}{c}{$\mathbf{2}$} & \multicolumn{1}{c}{19} & \multicolumn{1}{c}{ Literature $^{1}$} \\
\hline 5 & $4.53(\mathrm{dt}, J=7.3,5.8 \mathrm{~Hz}, 1 \mathrm{H})$ & $4.54(\mathrm{ddd}, J=8.0,5.3,2.8 \mathrm{~Hz}, 1 \mathrm{H})$ & $4.55(\mathrm{ddd}, J=8.0,5.3,2.8,1 \mathrm{H})$ \\
& & $2.34-2.27(\mathrm{~m}, 1 \mathrm{H})$ & $2.29(\mathrm{~m}, 1 \mathrm{H})$ \\
4 & $2.39-2.31(\mathrm{~m}, 1 \mathrm{H})$ & & $2.22(\mathrm{~m}, 1 \mathrm{H})$ \\
& $2.19-2.11(\mathrm{~m}, 1 \mathrm{H})$ & $2.26-2.18(\mathrm{~m}, 1 \mathrm{H})$ & \\
& & $2.66(\mathrm{ddd}, J=17.7,10.1,7.0,1 \mathrm{H})$ & $2.66(\mathrm{ddd}, J=16.7,10.1,7.0,1 \mathrm{H})$ \\
3 & $2.61-2.49(\mathrm{~m}, 2 \mathrm{H})$ & $2.47(\mathrm{ddd}, J=17.7,10.3,6.4,1 \mathrm{H})$ & $2.45(\mathrm{ddd}, J=16.7,10.3,6.4,1 \mathrm{H})$ \\
& & $4.97(\mathrm{dtd}, J=6.1,2.5,1.7 \mathrm{~Hz})$ & $4.97(\mathrm{~m}, 1 \mathrm{H})$ \\
$2^{\prime}$ & $4.85-4.81(\mathrm{~m}, 1 \mathrm{H})$ & $5.90(\mathrm{dt}, J=6.3,2.0 \mathrm{~Hz}, 1 \mathrm{H})$ & $5.92(\mathrm{br}, \mathrm{d}, J=9.3 \mathrm{~Hz}, 1 \mathrm{H})$ \\
$3^{\prime}$ & $5.96(\mathrm{dt}, J=6.3,1.8 \mathrm{~Hz}, 1 \mathrm{H})$ & $6.01(\mathrm{br}, \mathrm{d}, J=9.3 \mathrm{~Hz}, 1 \mathrm{H})$ \\
$4^{\prime}$ & $6.07(\mathrm{dt}, J=6.6,1.7 \mathrm{~Hz}, 1 \mathrm{H})$ & $6.01(\mathrm{dt}, J=6.3,2.0 \mathrm{~Hz}, 1 \mathrm{H})$ & $4.79(\mathrm{~m}, 1 \mathrm{H})$ \\
& & $4.79(\mathrm{dddd}, J=5.9,3.7,2.3,1.5$ & \\
$5^{\prime}$ & $4.74-4.71(\mathrm{~m}, 1 \mathrm{H})$ & $\mathrm{Hz}, 1 \mathrm{H})$ & $3.90(\mathrm{dq}, J=6.6,3.4 \mathrm{~Hz}, 1 \mathrm{H})$ \\
6 & $3.89(\mathrm{dq}, J=6.5,3.8 \mathrm{~Hz})$ & $3.90(\mathrm{dq}, J=6.5,3.4 \mathrm{~Hz})$ & $1.18(\mathrm{~d}, J=6.6 \mathrm{~Hz}, 1 \mathrm{H})$ \\
7 & $1.22(\mathrm{~d}, J=6.5 \mathrm{~Hz})$ & $1.17(\mathrm{~d}, J=6.5 \mathrm{~Hz})$ & $1.85, \mathrm{br}, \mathrm{s}$
\end{tabular}




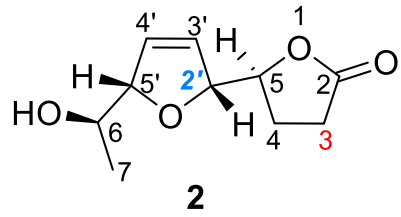

$\left(6 R, 5^{\prime} S, 2^{\prime} R, 5 S\right)$

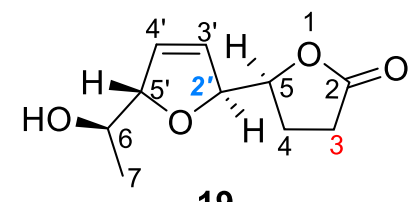

19

$\left(6 R, 5^{\prime} S, 2^{\prime} S, 5 S\right)$

\begin{tabular}{cccccc}
\hline Position & $\mathbf{2}$ & $\mathbf{1 9}$ & Literature $^{1}$ & $\Delta(\mathbf{2}$-Lit. $)$ & $\Delta$ (19-Lit.) \\
\hline 5 & 81.04 & 80.12 & 80.1 & +0.94 & +0.02 \\
4 & 23.54 & 23.75 & 23.7 & -0.16 & +0.05 \\
3 & 27.74 & 27.97 & 22.9 & +4.84 & +5.07 \\
& 176.39 & 177.24 & 177.2 & -0.85 & +0.04 \\
2 & 87.11 & 87.97 & 87.9 & -0.79 & +0.07 \\
3 & 129.50 & 128.79 & 128.7 & +0.80 & +0.09 \\
4 & 127.20 & 127.33 & 127.3 & -0.10 & +0.03 \\
5 & 91.00 & 91.01 & 90.9 & +0.10 & +0.11 \\
6 & 69.08 & 69.09 & 69.1 & -0.02 & -0.01 \\
7 & 18.92 & 17.94 & 17.9 & +1.02 & +0.04 \\
\hline
\end{tabular}

Dihydrofuran-2(3H)-one

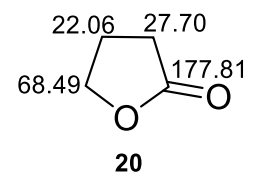

Liteature ${ }^{2}:{ }^{1} \mathrm{H}$ NMR (400 MHz, $\left.\mathrm{CDCl}_{3}\right): \delta 4.30(\mathrm{t}, \mathrm{J}=7.1 \mathrm{~Hz}, 2 \mathrm{H}), 2.45(\mathrm{t}, \mathrm{J}=7.9 \mathrm{~Hz}, 2 \mathrm{H}), 2.26$ -2.18 (m, $2 \mathrm{H})$.

${ }^{13} \mathrm{C} \mathrm{NMR}\left(100 \mathrm{MHz}, \mathrm{CDCl}_{3}\right): \delta 177.81,68.49,27.70,22.06$; 


\section{The Chemical Shifts of the $\mathbf{C} 3$ of $\gamma$-lactones Possessing Only One Substituent at C5}

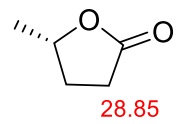

Synth. Commun. 48, 2801-2808

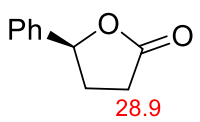

Angew. Chem. Int. Ed. 57, 1386-1389.

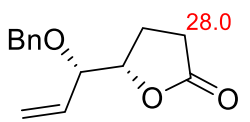

Tetrahedron, 67, 9358-9367

$\mathrm{HOH}_{2} \mathrm{C}$<smiles>C/C=C/C=C\[C@H](O)[C@@H]1C=[18CH]CC1</smiles>

J. Agric. Food Chem. 64, 217-225

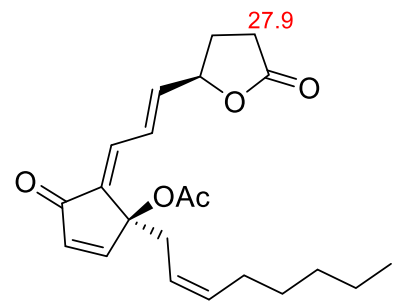<smiles>O=C1CO[C@@H](C(=O)O)C1</smiles><smiles>O=C1CO[C@H](CO)C1</smiles>

J. Org. Chem. 75, 2820-2835<smiles>O=C1CO[C@@H](Cc2ccc(O)c(O)c2)C1</smiles>

Synthesis, 9, 1512-1520<smiles>C/C=C/C=C\[C@@H](O)[C@H]1C[C@@H](C)C(=O)O1</smiles>
J. Nat. Prod. 62, 253-256

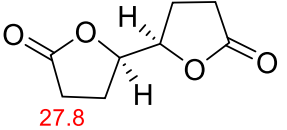

Tetrahedron, 65, 396-399<smiles>C[C@@H]1C[C@H]([C@@H](C)O)C(=O)O1</smiles>

Tetrahedron, 55,1043-1056<smiles></smiles><smiles>C/C=C(\C)C(=O)O[C@H](/C=C/[C@H](O)[C@H](C)O)[C@H]1CC([18F])C(=O)O1</smiles>

Eur. J. Org. Chem. 31, 6891-6899

Synlett, 5, 779-782

Org. Biomol. Chem. 13, 4051-4058<smiles></smiles>

J. Nat. Prod. 69, 980-982<smiles>O=C1O[C@H]([C@H]2CC[C@@H]3CCCC(O)N32)C[C@H]1[18OH]</smiles>

Org. Lett. 12, 5752-5755<smiles>C/C=C/C(=O)C[C@H]1C(=O)C=C[C@]1(O)CCCC[C@H]1C[C@@H](Br)C(=O)O1</smiles><smiles>C[C@@H]1C[C@H](C/C=C\[C@H](O)[C@@H](C)O)C(=O)O1</smiles>

J. Nat. Prod. 71, 701-705

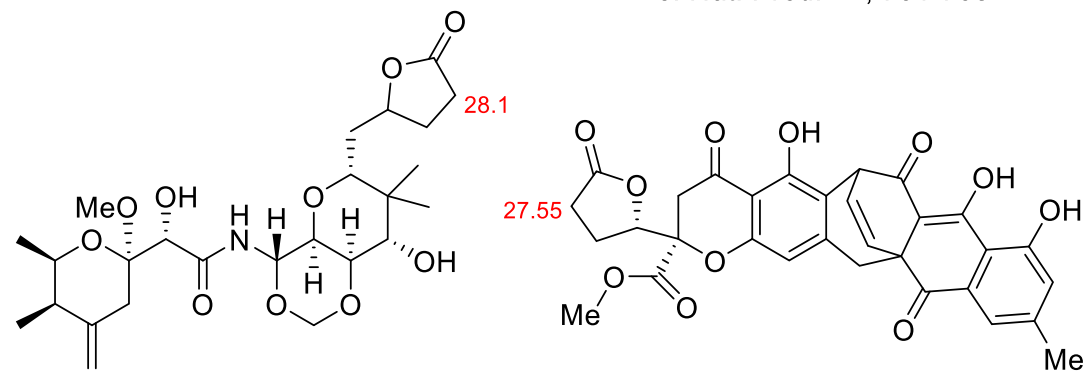

J. Am. Chem. Soc. 115, 8558-8564

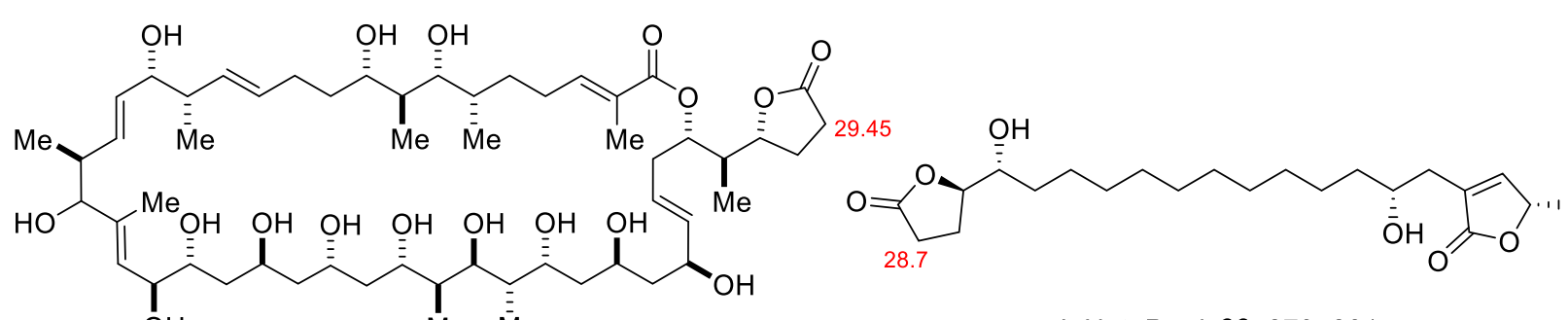

Angew. Chem. Int. Ed. 46, 545-548. 


\section{Comments about Literature Misassignment}

The absolute stereochemistry of C6, i.e., (R) is determined by the Mosher's method in the initial report. ${ }^{1}$ The absolute stereochemistry of the other chiral centers are assigned in 2017 by the chiroptical methods. ${ }^{3}$ In addition, D. corticola produces several metabolites: furanones such as sapinofuranone $\mathrm{B}$, sapinofuranone $\mathrm{C}$, diplofuranones $\mathrm{A}$, and diplobifuranylones $\mathrm{A}-\mathrm{C}$. All the known structures have lactone rings with $S$ configuration at the C5 position. So, the C6 and C5 configurations, being $R$ and $S$, respectively, are secure.

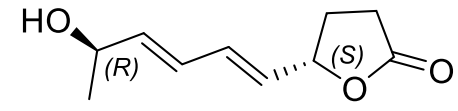

diplofuranone A

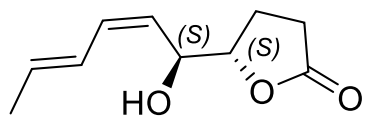

sapinofuranone $B$

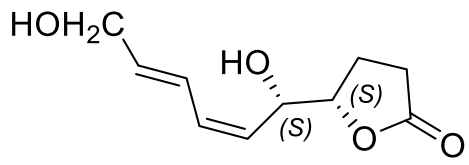

sapinofuranone $\mathrm{C}$

The only other potential stereoisomers are $\left(6 R, 5^{\prime} R, 2^{\prime} R, 5 S\right)$ and $\left(6 R, 5^{\prime} R, 2^{\prime} S, 5 S\right)$. Based on the coupling constant between $\mathrm{H}-2$ ' and $\mathrm{H}_{-} 5^{\prime}$ reported in the initial report, ${ }^{1}$ the relative stereochemistry of the dihydrofuran moiety should be trans. In this way, only two possible stereoisomers are left: $\left(6 R, 5^{\prime} S, 2^{\prime} S, 5 S\right)$ and $\left(6 R, 5^{\prime} R, 2^{\prime} R, 5 S\right)$. It is reasonable that ${ }^{1} \mathrm{H}$ and ${ }^{13} \mathrm{C}$ NMR spectra should be able to rule them out.

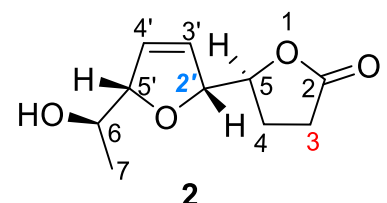

(6R, 5'S, 2'R, 5S)

cis

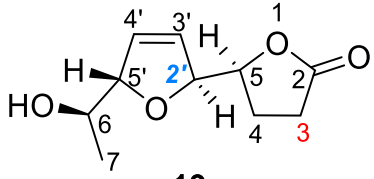

19

(6R, 5'S, 2'S, 5S)

trans

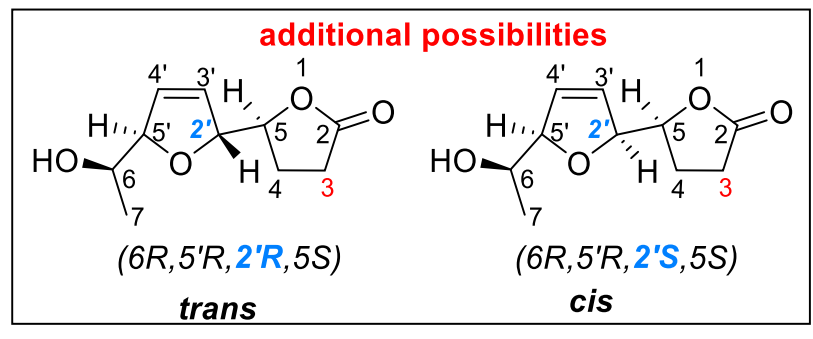




\section{Computational Details.}

Structural optimizations were performed at the B3LYP ${ }^{4-5}$ level of theory with cc-pVDZ basis set.

The Cartesian coordinates of $\mathbf{1 9}$, the revised structure of diplobifuranylone B with a transdihydrofuran ring.

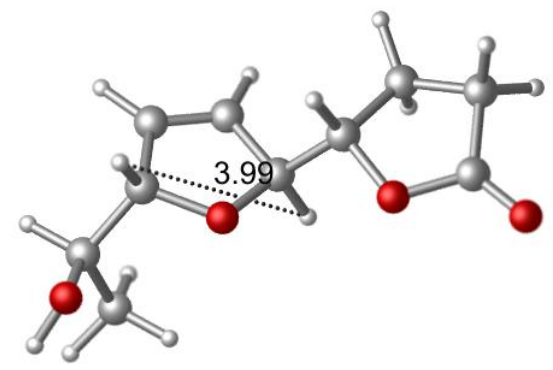
$\begin{array}{llll}\text { C } & 0.616300 & 1.830200 & 0.255100\end{array}$
$\begin{array}{llll}\text { C } & 1.797000 & 1.714200 & -0.354800\end{array}$
$\begin{array}{llll}\text { C } & 2.074300 & 0.271100 & -0.682300\end{array}$
C $\quad-0.023500 \quad 0.468600 \quad 0.382100$
$\begin{array}{llll}\mathrm{H} & 0.159700 & 2.752400 & 0.616000\end{array}$
H $\quad 2.484600 \quad 2.527000 \quad-0.593900$
$\begin{array}{lllll}\text { O } & 0.929600 & -0.435900 & -0.179400\end{array}$
H $\quad-0.207900 \quad 0.191000 \quad 1.440100$
$\mathrm{H} \quad 2.144800 \quad 0.108700 \quad-1.775900$
$\begin{array}{llll}\text { C } & 3.384700 & -0.280800 & -0.092300\end{array}$
H $\quad 4.175400 \quad 0.464200 \quad-0.331700$
C $\quad-1.362500 \quad 0.355800 \quad-0.350900$
$\begin{array}{llll}\text { C } & -2.438200 & 1.359500 & 0.102500\end{array}$
$\mathrm{H} \quad-1.174400 \quad 0.426700 \quad-1.436600$
$\begin{array}{llll}\text { C } & -3.291500 & -0.878000 & 0.033400\end{array}$ 


$\begin{array}{lrrr}\mathrm{C} & -3.731400 & 0.575100 & -0.119200 \\ \mathrm{H} & -2.383700 & 2.305000 & -0.454500 \\ \mathrm{H} & -2.309400 & 1.585900 & 1.174800 \\ \mathrm{H} & -4.122200 & 0.701600 & -1.143600 \\ \mathrm{H} & -4.547800 & 0.802700 & 0.578600 \\ \mathrm{C} & 3.326800 & -0.491100 & 1.417400 \\ \mathrm{H} & 4.295000 & -0.865000 & 1.791400 \\ \mathrm{H} & 3.108500 & 0.450900 & 1.945900 \\ \mathrm{H} & 2.544600 & -1.224800 & 1.660100 \\ \mathrm{O} & 3.639000 & -1.497400 & -0.788800 \\ \mathrm{H} & 4.386800 & -1.934400 & -0.357100 \\ \mathrm{O} & -1.931800 & -0.947000 & -0.087300 \\ \mathrm{O} & -3.970100 & -1.849700 & 0.223300\end{array}$

The Cartesian coordinates of $\mathbf{2}$, the norminal structure of diplobifuranylone B with a cisdihydrofuran ring. It has an intramolecular H-bond.

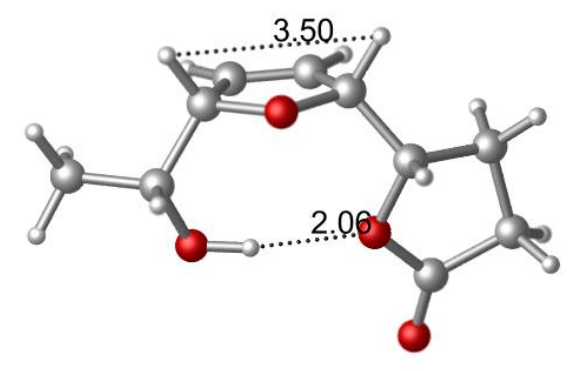
C $\quad-0.323600 \quad-1.428000 \quad 1.197200$
C $\quad-1.580100 \quad-0.983500 \quad 1.230000$
C $\quad-2.102400 \quad-0.778600 \quad-0.166600$
C $\quad 0.125600 \quad-1.579800 \quad-0.235300$
H $\quad 0.299300 \quad-1.667300 \quad 2.059400$ 


$$
\begin{array}{lrrr}
\mathrm{H} & -2.167300 & -0.780900 & 2.125400 \\
\mathrm{O} & -0.989200 & -1.140800 & -1.013600 \\
\mathrm{H} & -2.944900 & -1.463700 & -0.391900 \\
\mathrm{C} & -2.545000 & 0.662100 & -0.496100 \\
\mathrm{H} & -2.606600 & 0.707400 & -1.604400 \\
\mathrm{C} & 1.358700 & -0.775800 & -0.653600 \\
\mathrm{C} & 2.692200 & -1.188500 & -0.008300 \\
\mathrm{H} & 1.420900 & -0.819000 & -1.754800 \\
\mathrm{C} & 2.368600 & 1.190600 & 0.090900 \\
\mathrm{C} & 3.463600 & 0.132800 & 0.025400 \\
\mathrm{H} & 3.193800 & -1.983800 & -0.576800 \\
\mathrm{H} & 2.524300 & -1.558200 & 1.016000 \\
\mathrm{H} & 4.033800 & 0.304100 & -0.903900 \\
\mathrm{H} & 4.158200 & 0.251600 & 0.867100 \\
\mathrm{C} & -3.914700 & 0.990500 & 0.086300 \\
\mathrm{H} & -4.185500 & 2.024600 & -0.173400 \\
\mathrm{H} & -4.691800 & 0.316200 & -0.307300 \\
\mathrm{H} & -3.903200 & 0.916700 & 1.186100 \\
\mathrm{O} & -1.638100 & 1.625300 & 0.002000 \\
\mathrm{O} & 1.180100 & 0.617000 & -0.293300 \\
\mathrm{O} & 2.446500 & 2.345400 & 0.400800 \\
\mathrm{H} & 0.351600 & -2.641300 & -0.477800 \\
\mathrm{H} & -0.734000 & 1.368200 & -0.250500 \\
& & & \\
\hline
\end{array}
$$




\section{Reference}

1. Evidente, A.; Andolfi, A.; Fiore, M.; Spanu, E.; Maddau, L.; Franceschini, A.; Marras, F.; Motta, A. Diplobifuranylones A and B, 5'-Monosubstituted Tetrahydro-2H-bifuranyl-5-ones Produced by Diplodia corticola, a Fungus Pathogen of Cork Oak. J. Nat. Prod. 2006, 69, 671-674.

2. Das, A.; Chaudhuri, R.; Liu, R.-S. Gold-Catalyzed Oxidative Cleavage of Aryl-Substituted Alkynyl Ethers Using Molecular Oxygen. Simultaneous Degradation of C-H and Single and Triple Carbon-Carbon Bonds Under Ambient Conditions. Chem. Comm. 2009, 4046-4048.

3. Mazzeo, G.; Cimmino, A.; Masi, M.; Longhi, G.; Maddau, L.; Memo, M.; Evidente, A.; Abbate, S. Importance and Difficulties in the Use of Chiroptical Methods to Assign the Absolute Configuration of Natural Products: The Case of Phytotoxic Pyrones and Furanones Produced by Diplodia Corticola. J. Nat. Prod. 2017, 80, 2406-2415.

4. Becke, A. D. Density-Functional Thermochemistry. III. The Role of Exact Exchange. $J$. Chem. Phys. 1993, 98, 5648-5652.

5. Lee, C.; Yang, W.; Parr, R. G. Development of the Colle-Salvetti Correlation-Energy Formula into a Functional of the Electron Density. Physical Review B. 1988, 37, 785-789. 


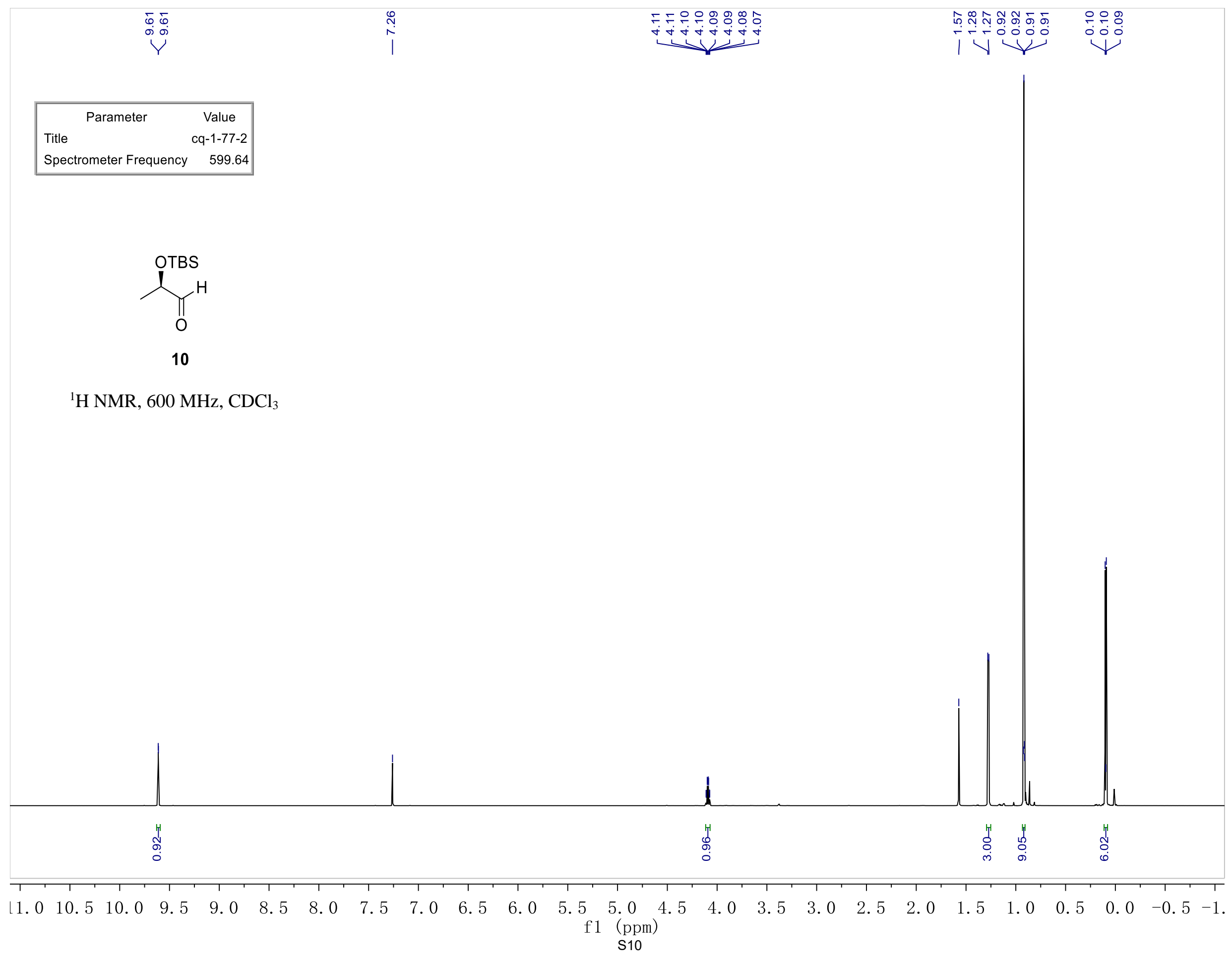




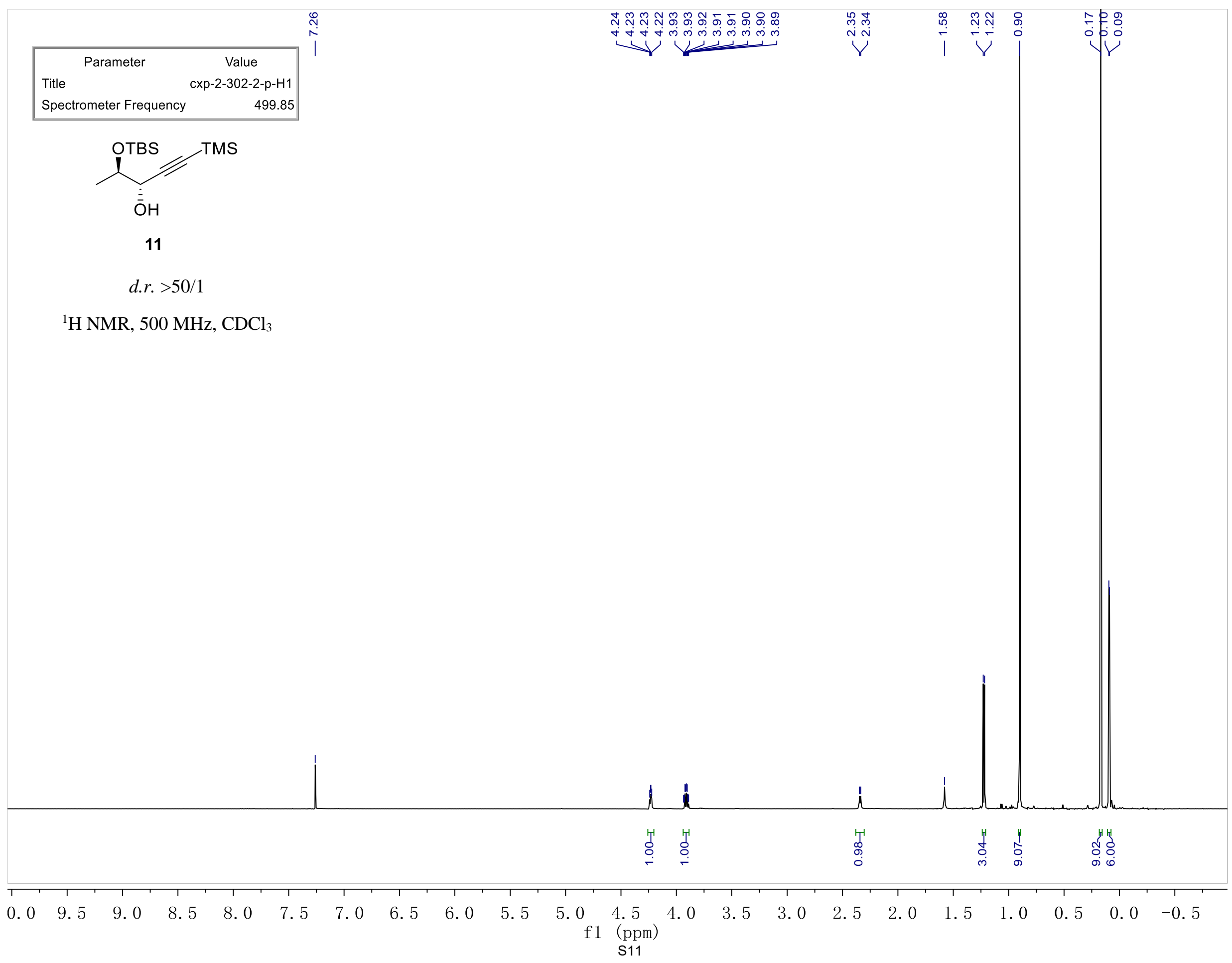




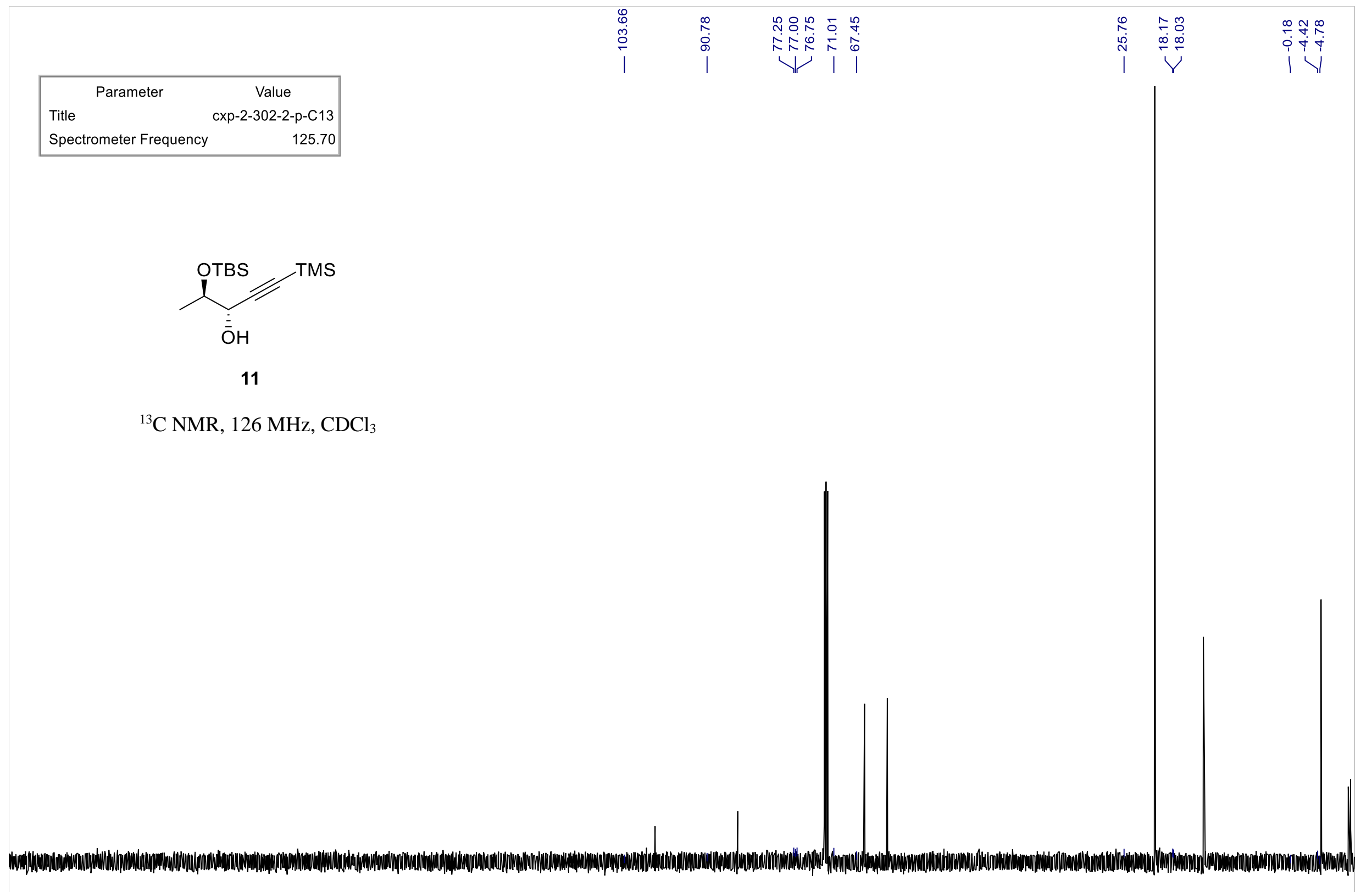

\begin{tabular}{|c|c|c|c|c|c|c|c|c|c|c|c|c|c|c|c|c|c|c|c|}
\hline 190 & 180 & 170 & 160 & 150 & 140 & 130 & 120 & 110 & $\begin{array}{r}100 \\
\text { f1 }\end{array}$ & $\begin{array}{r}1 \\
90 \\
\text { (ppm) } \\
\text { S12 }\end{array}$ & 80 & 70 & 60 & 50 & 40 & 30 & 20 & 10 & 0 \\
\hline
\end{tabular}




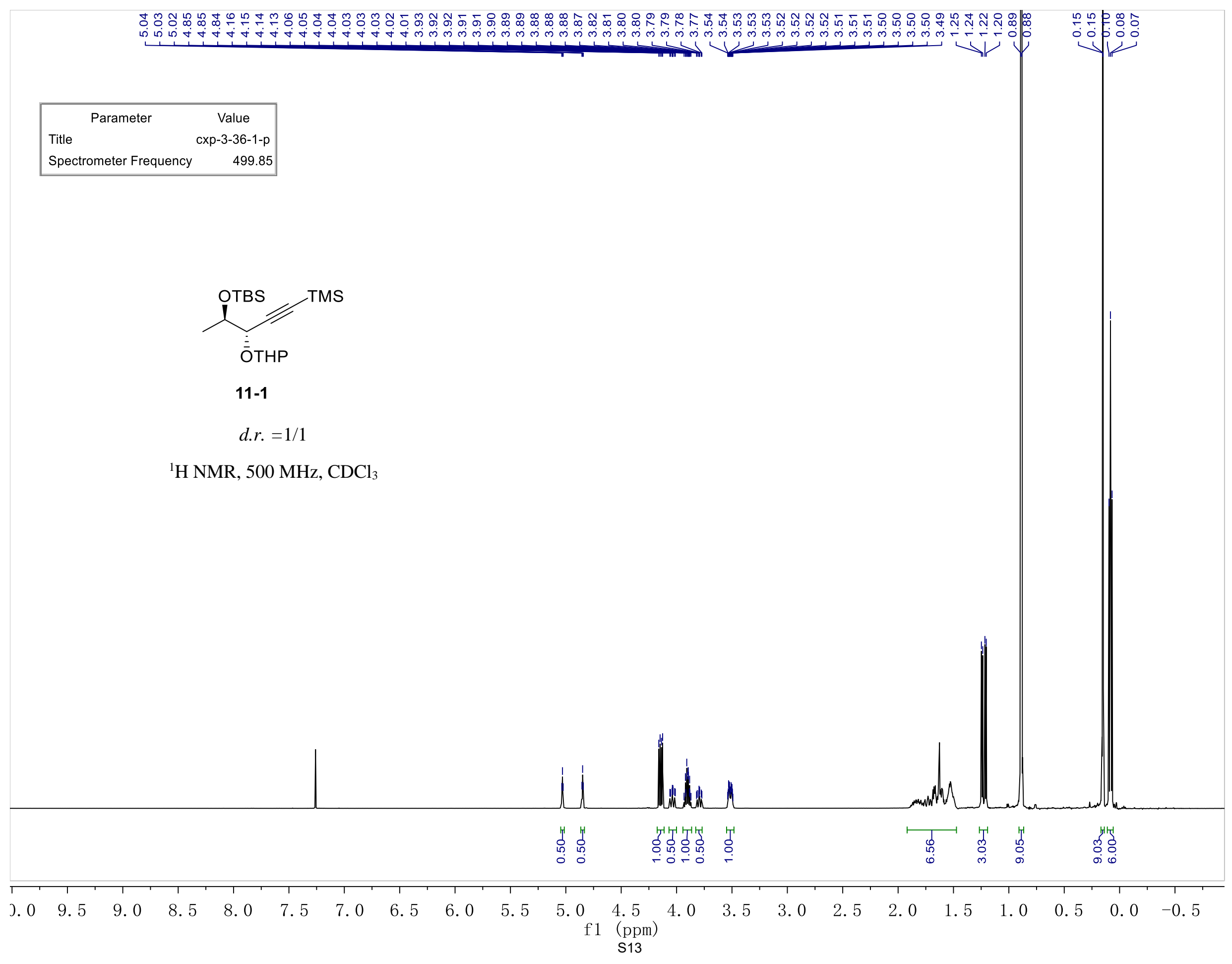




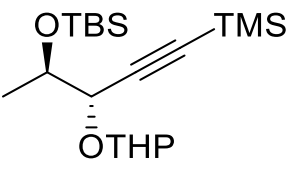

11-1

${ }^{13} \mathrm{C} \mathrm{NMR}, 126 \mathrm{MHz}, \mathrm{CDCl}_{3}$

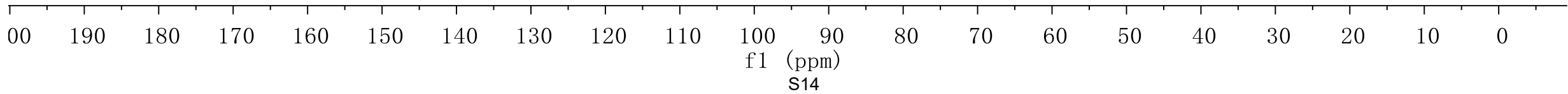




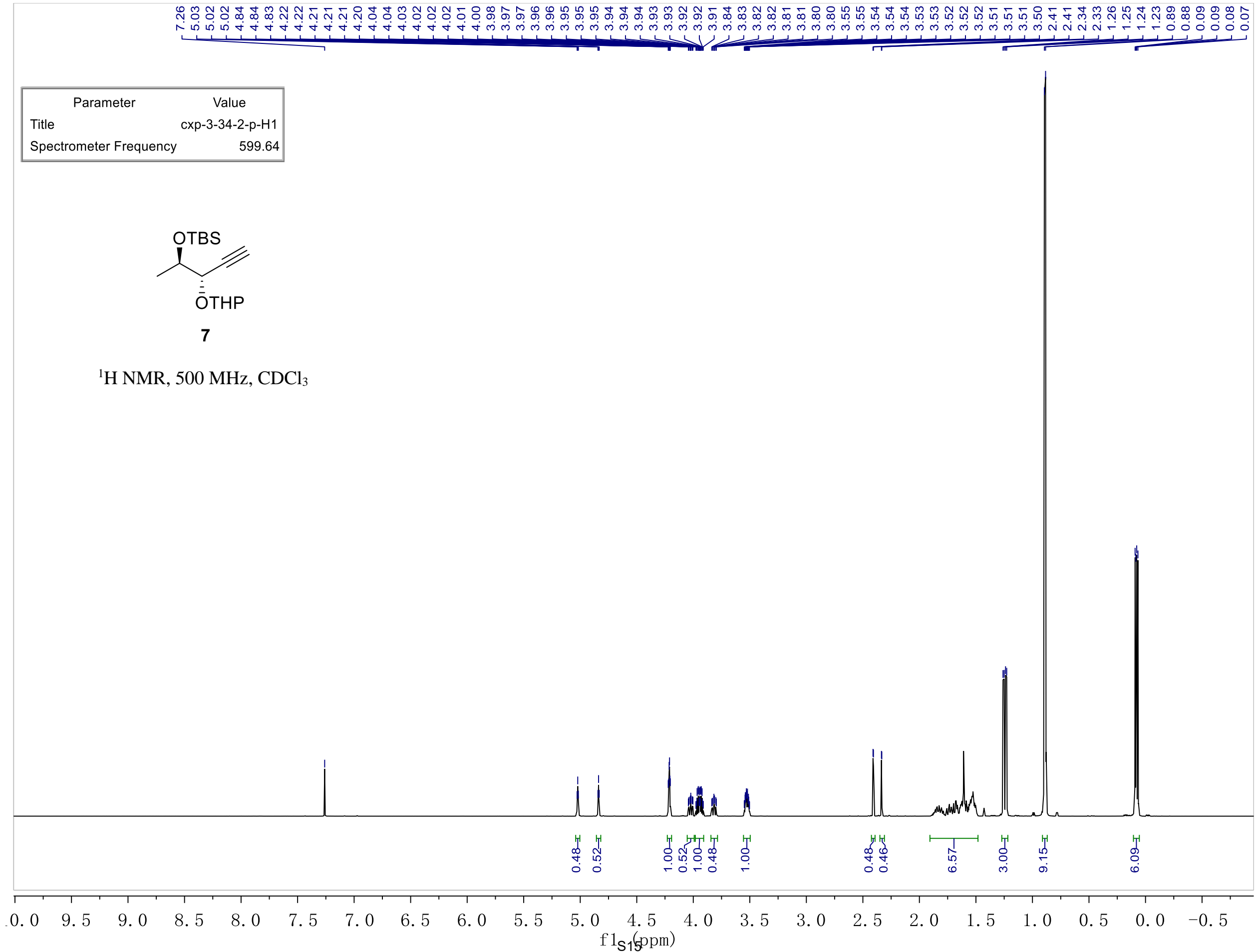




\begin{tabular}{|lr|}
\hline \multicolumn{1}{c}{ Parameter } & \multicolumn{1}{c|}{ Value } \\
Title & cxp-3-34-2-p-C13 \\
Spectrometer Frequency & 125.70 \\
\hline
\end{tabular}

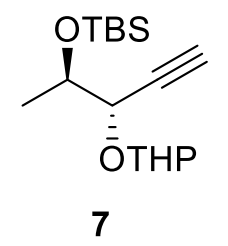

${ }^{13} \mathrm{C} \mathrm{NMR}, 126 \mathrm{MHz}, \mathrm{CDCl}_{3}$

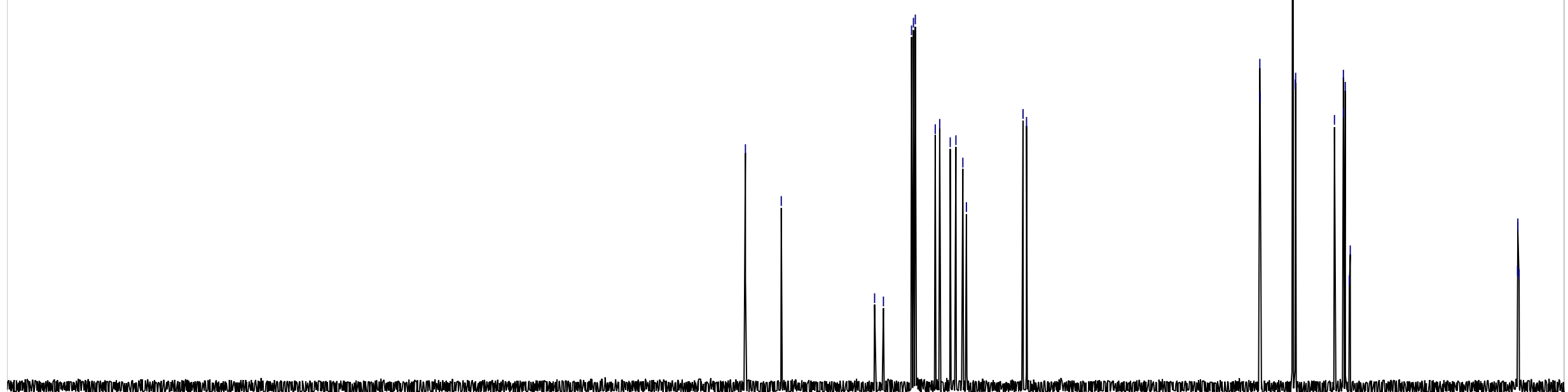

\begin{tabular}{|c|c|c|c|c|c|c|c|c|c|c|c|c|c|c|c|c|c|c|c|}
\hline$T$ & $T$ & $T$ & $T$ & $T$ & $T$ & $T$ & $T$ & $T$ & $T$ & $T$ & $T$ & $T$ & $T$ & $T$ & $T$ & $T$ & $T$ & $T$ & $T$ \\
\hline 190 & 180 & 170 & 160 & 150 & 140 & 130 & 120 & 110 & $\begin{array}{r}100 \\
\mathrm{f} 1\end{array}$ & $\begin{array}{c}90 \\
(\mathrm{ppm})\end{array}$ & 80 & 70 & 60 & 50 & 40 & 30 & 20 & 10 & 0 \\
\hline
\end{tabular}




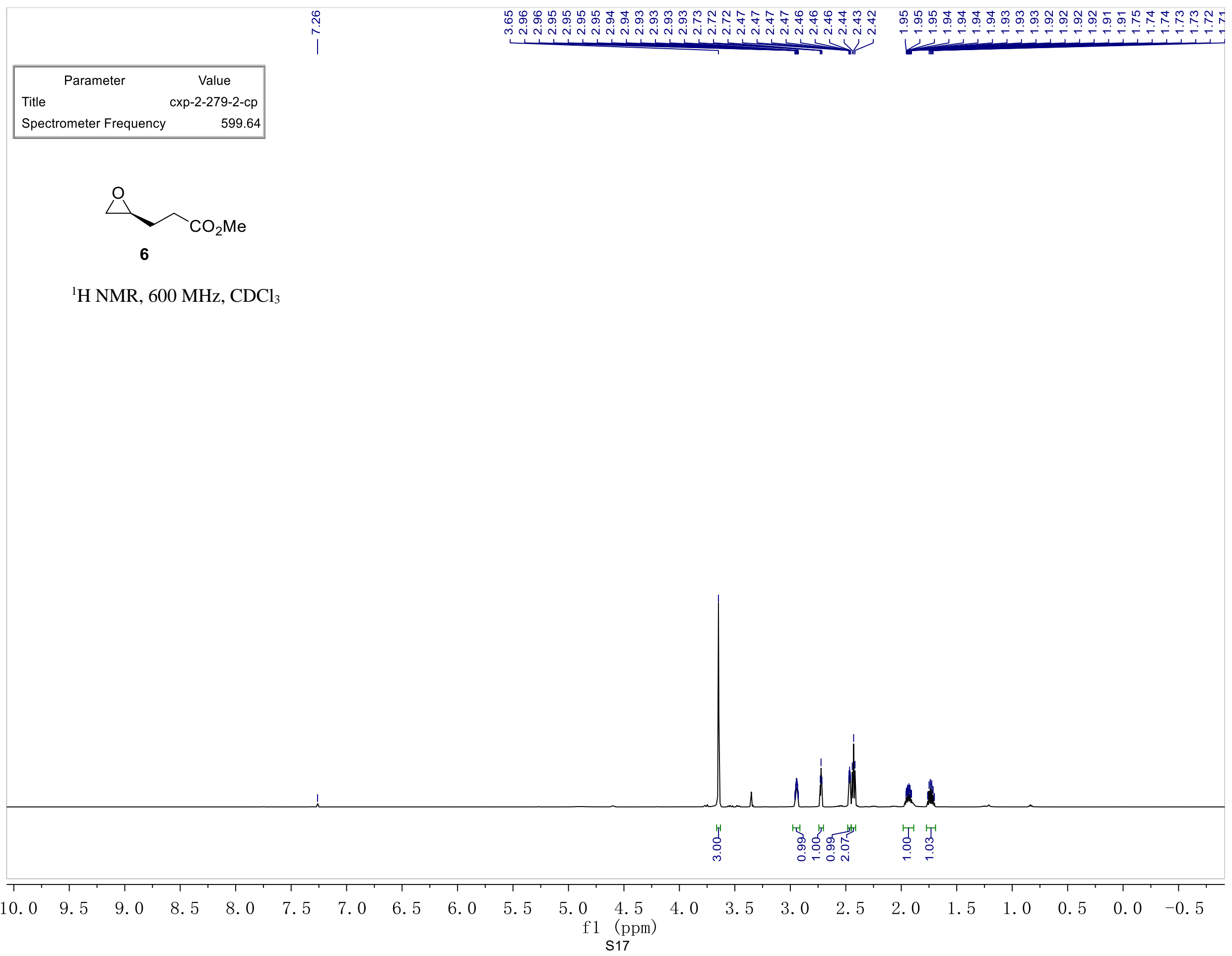




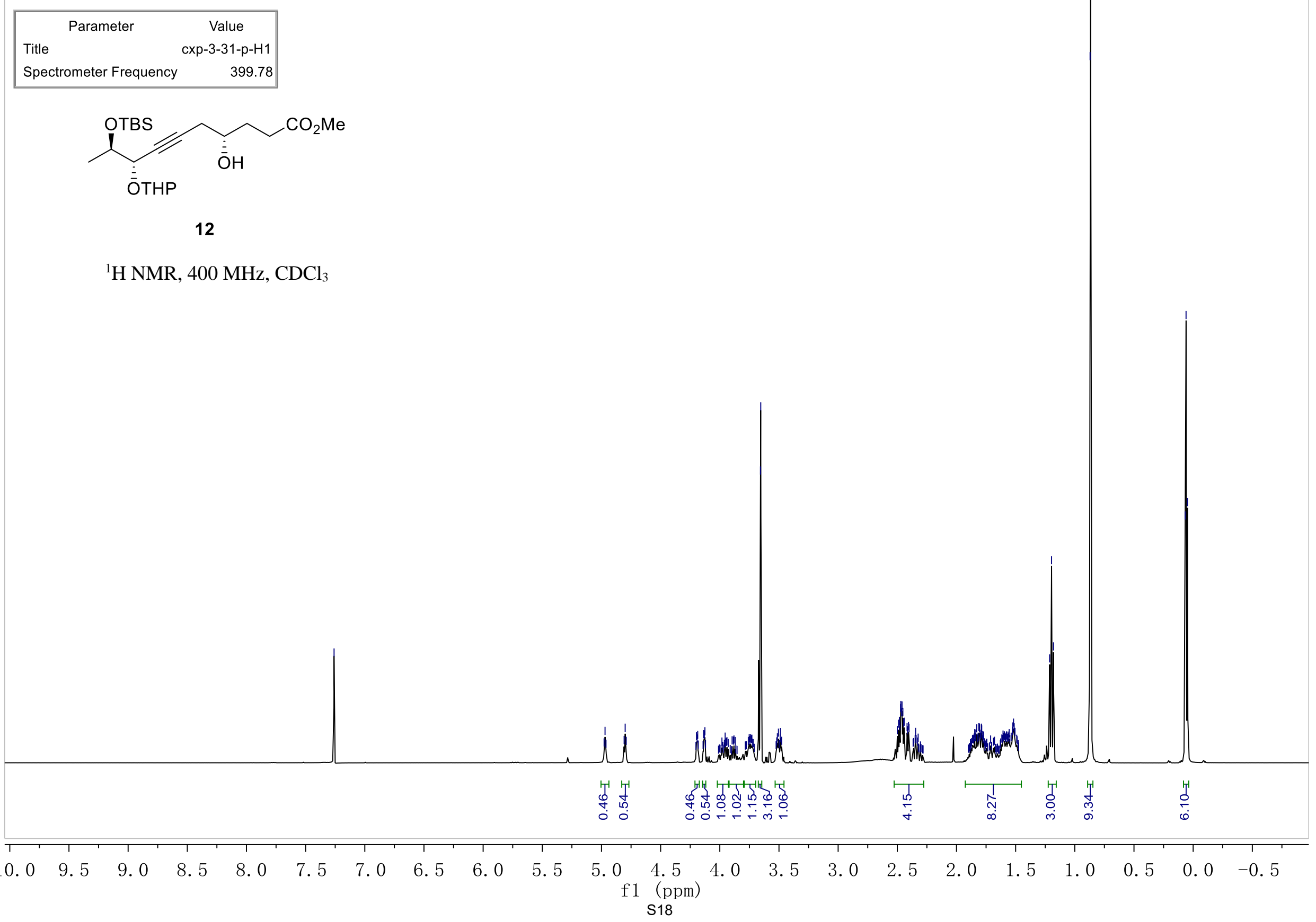



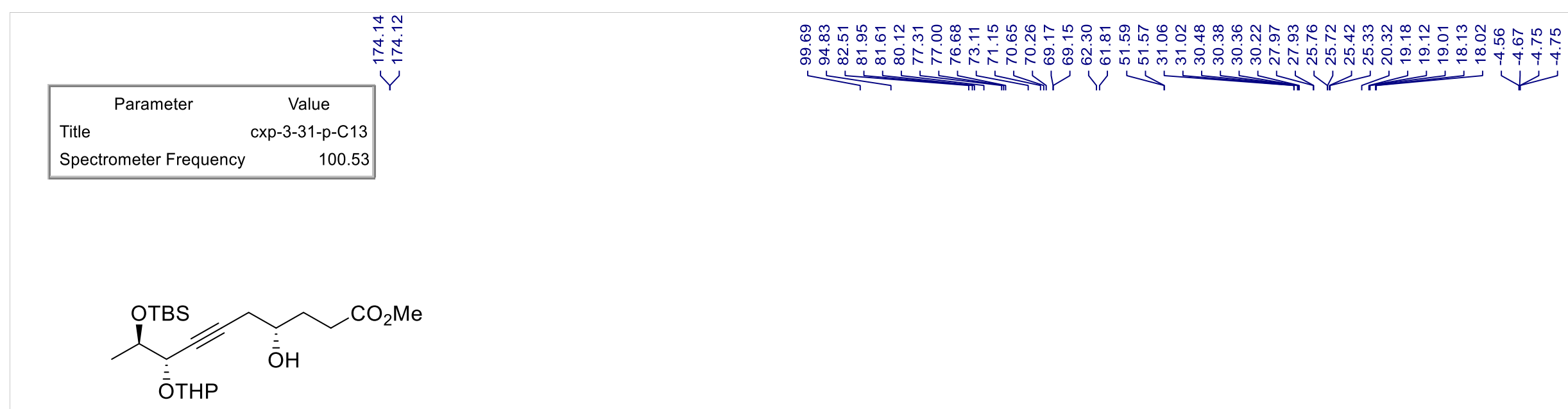

12

${ }^{13} \mathrm{C} \mathrm{NMR,} 101 \mathrm{MHz}, \mathrm{CDCl}_{3}$

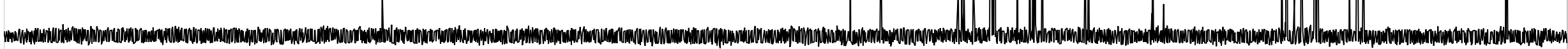

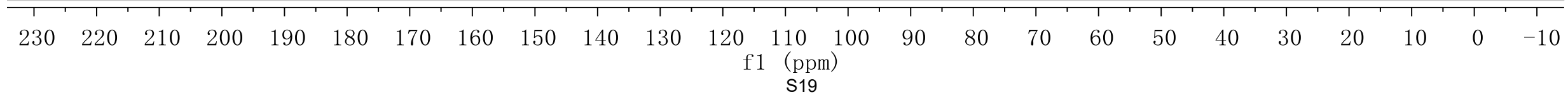




\begin{tabular}{|lr|}
\hline \multicolumn{1}{|c|}{ Parameter } & \multicolumn{1}{c|}{ Value } \\
Title & cxp-3-32-p-H1 \\
Spectrometer Frequency & 599.64 \\
\hline
\end{tabular}

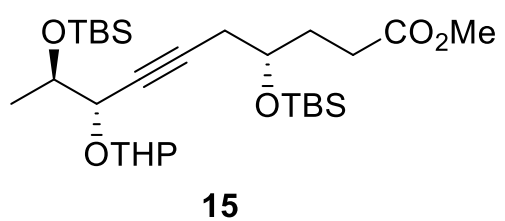

${ }^{1} \mathrm{H} \mathrm{NMR}, 600 \mathrm{MHz}, \mathrm{CDCl}_{3}$

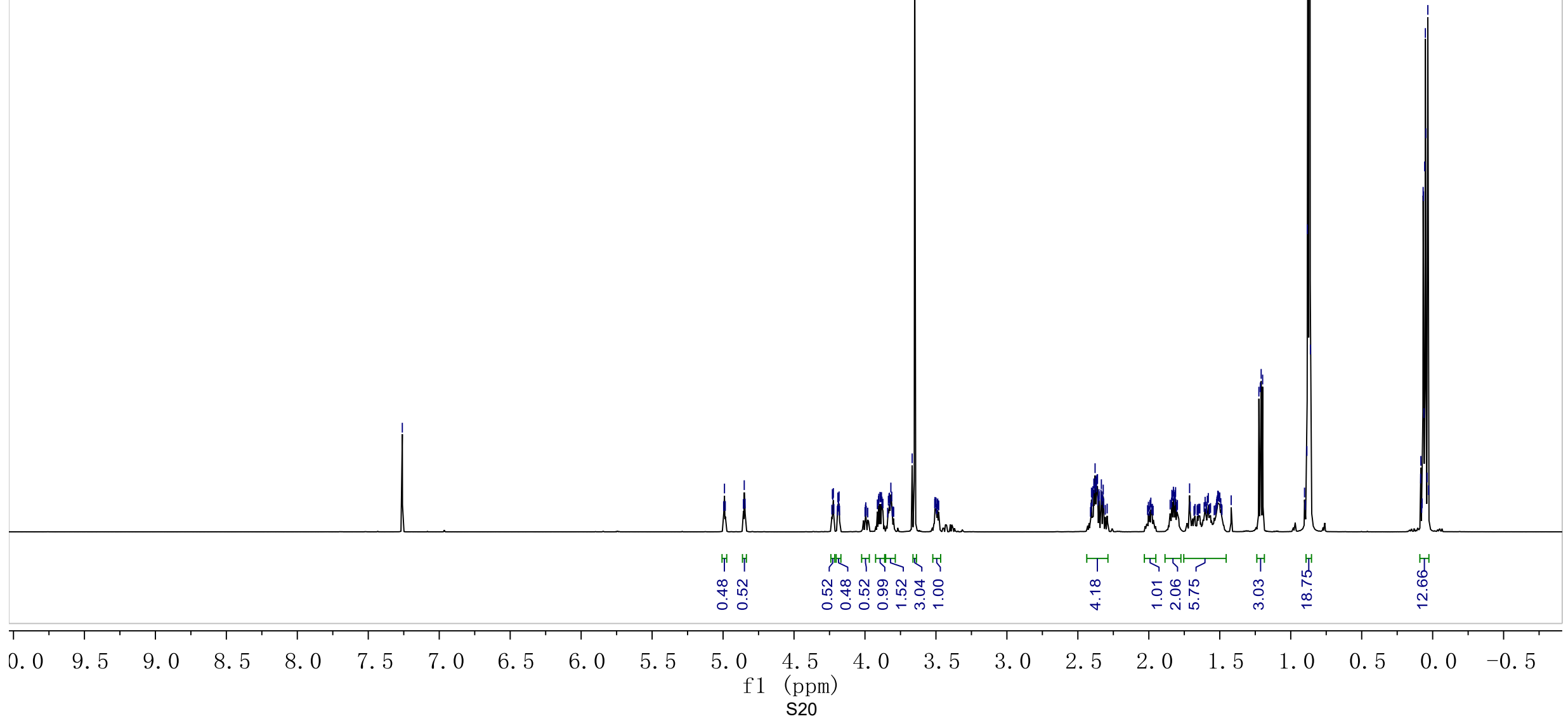



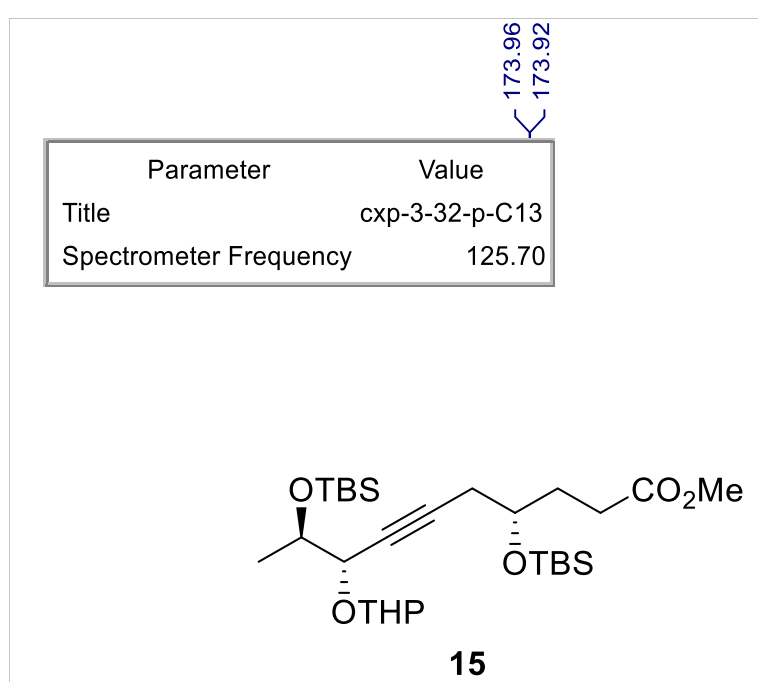

${ }^{13} \mathrm{C} \mathrm{NMR}, 126 \mathrm{MHz}, \mathrm{CDCl}_{3}$

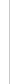

\begin{tabular}{|c|c|c|c|c|c|c|c|c|c|c|c|c|c|c|c|c|c|c|c|c|c|c|c|}
\hline 1 & 1 & 1 & 1 & 1 & 1 & 1 & 1 & $T$ & 1 & 1 & 1 & 21 & $T$ & 1 & 1 & $T$ & $T$ & $T$ & $T$ & $T$ & 1 & 1 & 1 \\
\hline 220 & 210 & 200 & 190 & 180 & 170 & 160 & 150 & 140 & 130 & 120 & $\begin{array}{l}110 \\
\mathrm{f} 1\end{array}$ & $\begin{array}{l}100 \\
\left(\begin{array}{l}10 p m) \\
\text { s21 }\end{array}\right.\end{array}$ & 90 & 80 & 70 & 60 & 50 & 40 & 30 & 20 & 10 & 0 & -10 \\
\hline
\end{tabular}




\begin{tabular}{|lr|}
\hline \multicolumn{1}{|c|}{ Parameter } & \multicolumn{1}{c|}{ Value } \\
Title & cxp-3-19-p \\
Spectrometer Frequency & 499.85 \\
\hline
\end{tabular}

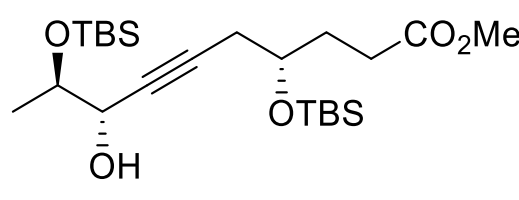

16

${ }^{1} \mathrm{H} \mathrm{NMR}, 600 \mathrm{MHz}, \mathrm{CDCl}_{3}$

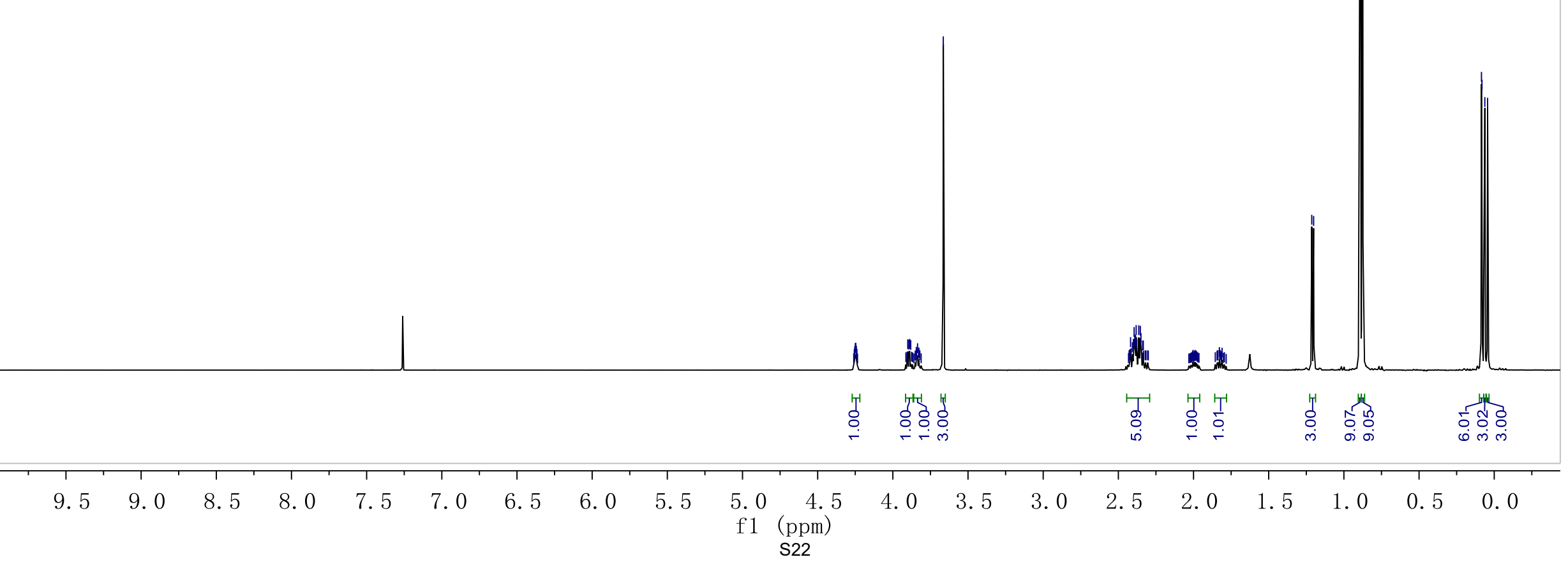




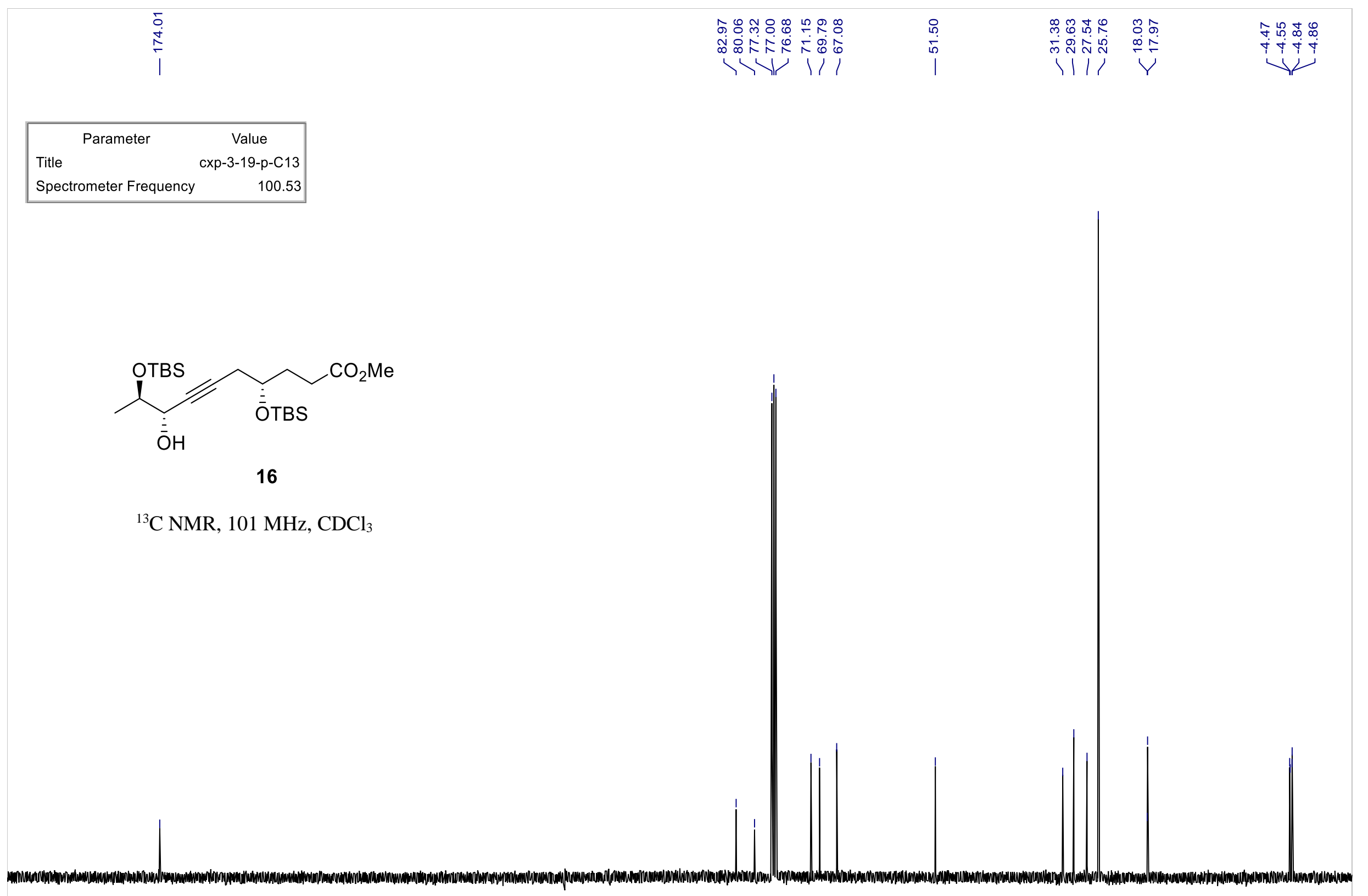

\begin{tabular}{|c|c|c|c|c|c|c|c|c|c|c|c|c|c|c|c|c|c|c|c|c|}
\hline 1 & 1 & 1 & 1 & 1 & 1 & 1 & 1 & 1 & 1 & 1 & $T$ & 1 & $T$ & 1 & $T$ & $T$ & $T$ & $T$ & $T$ & 1 \\
\hline 190 & 180 & 170 & 160 & 150 & 140 & 130 & 120 & 110 & $\begin{array}{l}100 \\
f 1\end{array}$ & $\begin{array}{l}90 \\
(\mathrm{ppm})\end{array}$ & 80 & 70 & 60 & 50 & 40 & 30 & 20 & 10 & 0 & -10 \\
\hline
\end{tabular}




\begin{tabular}{|lr|}
\hline \multicolumn{1}{|c|}{ Parameter } & \multicolumn{1}{c|}{ Value } \\
Title & cxp-3-41-p-H1 \\
Spectrometer Frequency & 599.64 \\
\hline
\end{tabular}

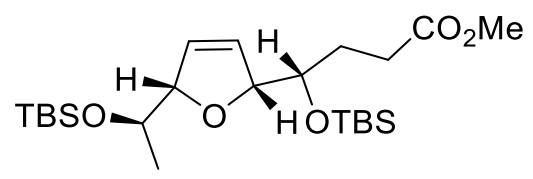

17

d.r. $=95 / 5$

${ }^{1} \mathrm{H} \mathrm{NMR}, 600 \mathrm{MHz}, \mathrm{CDCl}_{3}$

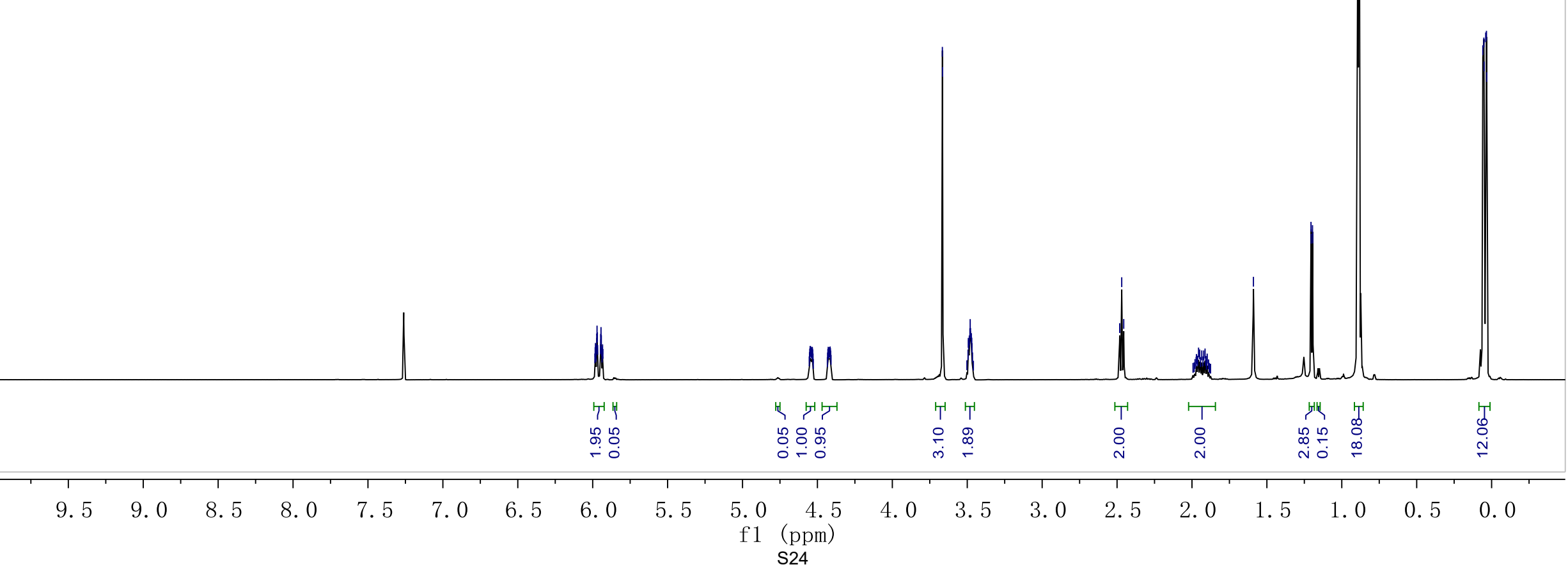




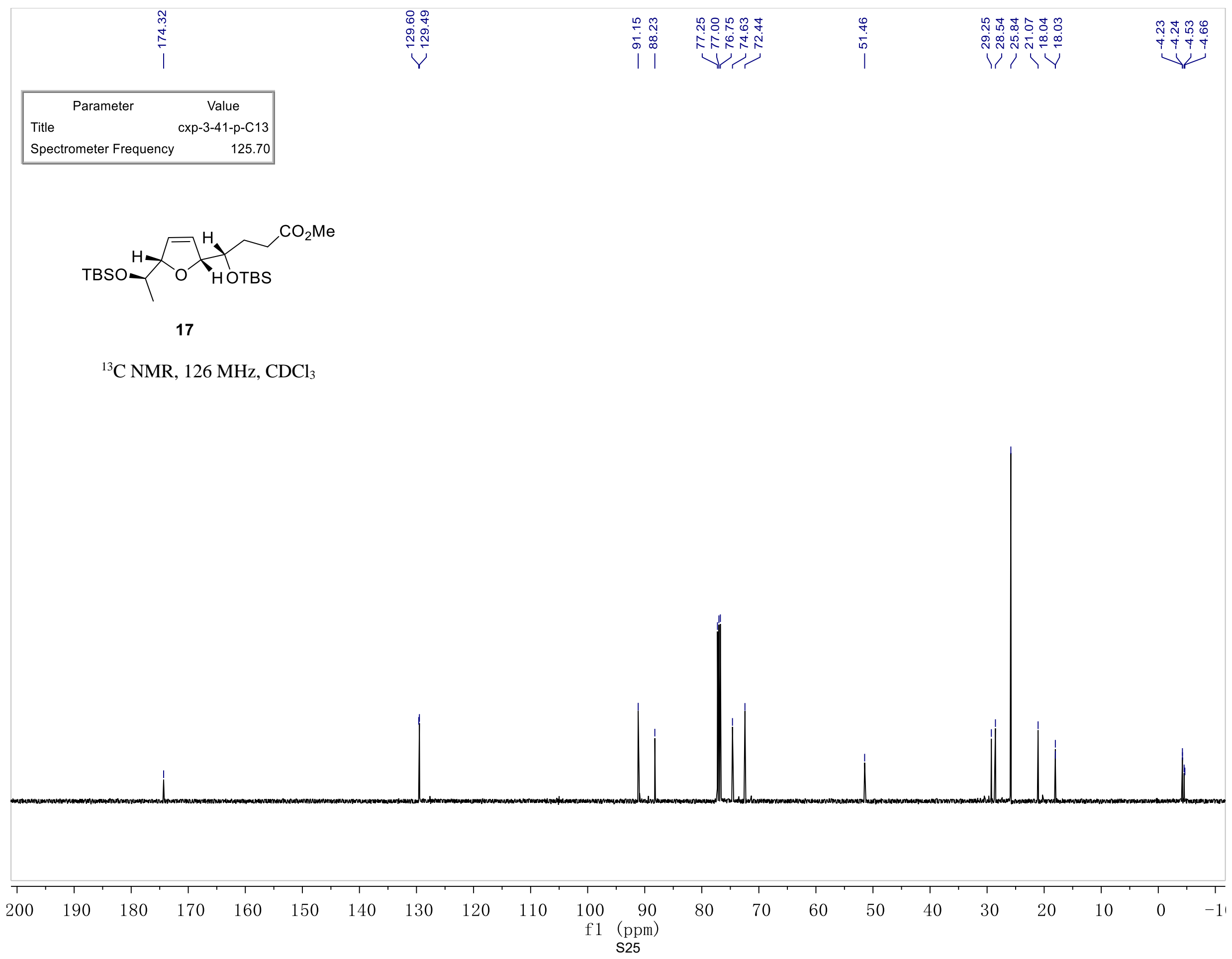




\begin{tabular}{|lr|}
\hline \multicolumn{1}{|c|}{ Parameter } & \multicolumn{1}{c|}{ Value } \\
Title & cxp-3-39-2-p-H1 \\
Spectrometer Frequency & 599.64 \\
\hline
\end{tabular}

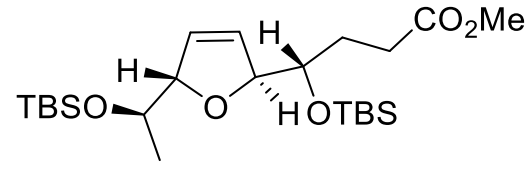

18

d.r. $=95 / 5$

${ }^{1} \mathrm{H}$ NMR, $600 \mathrm{MHz}, \mathrm{CDCl}_{3}$

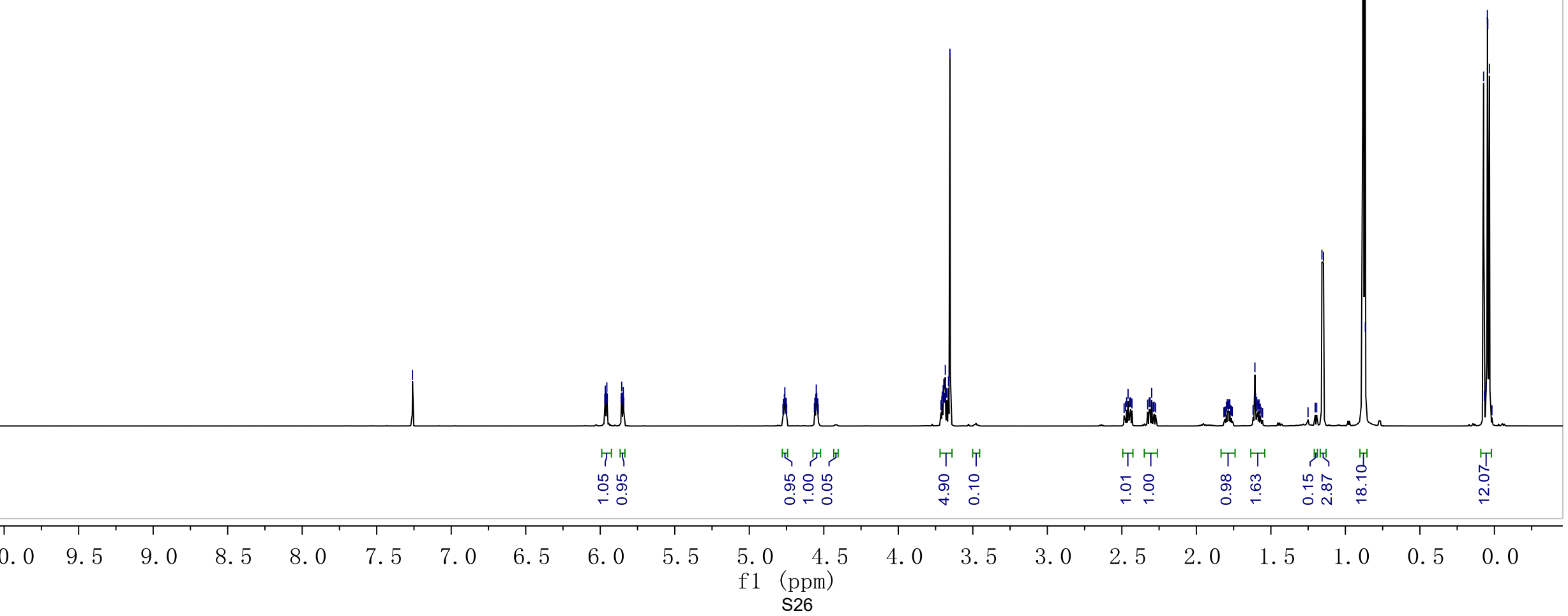




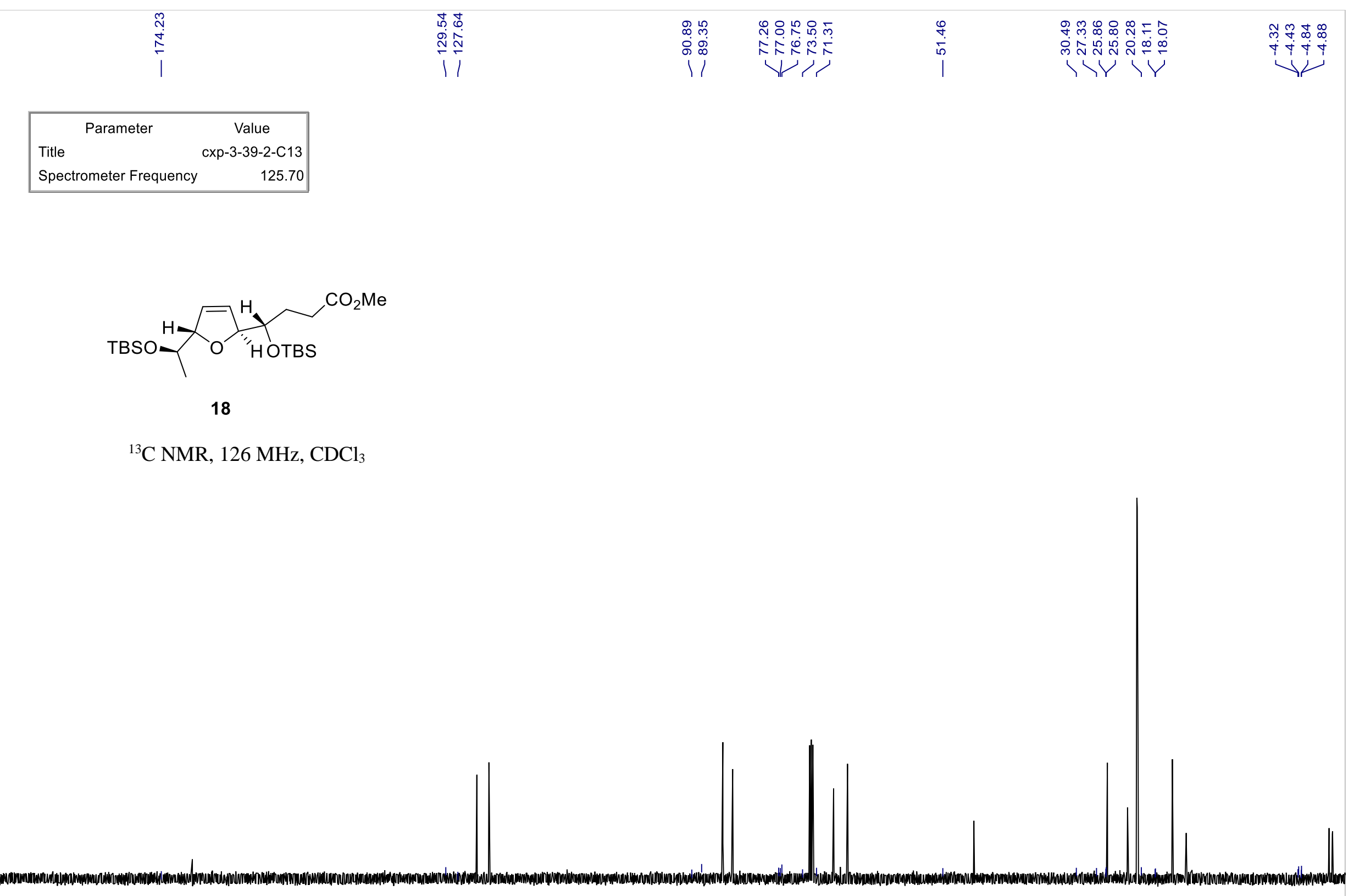

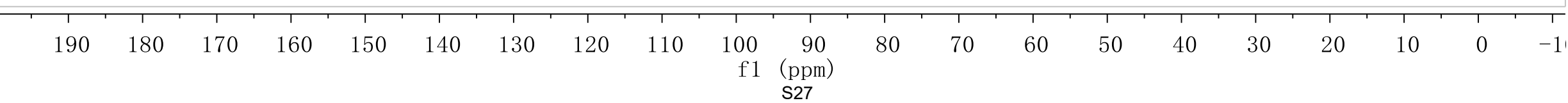



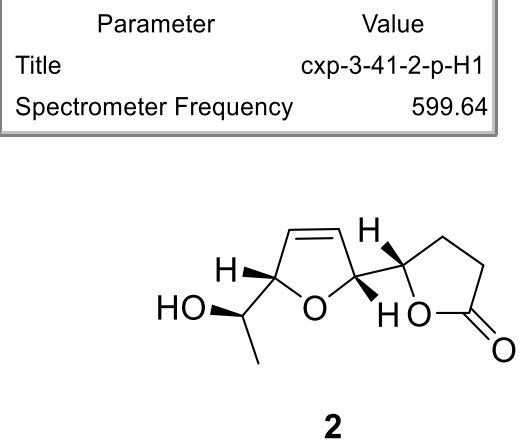

${ }^{1} \mathrm{H} \mathrm{NMR}, 600 \mathrm{MHz}, \mathrm{CDCl}_{3}$

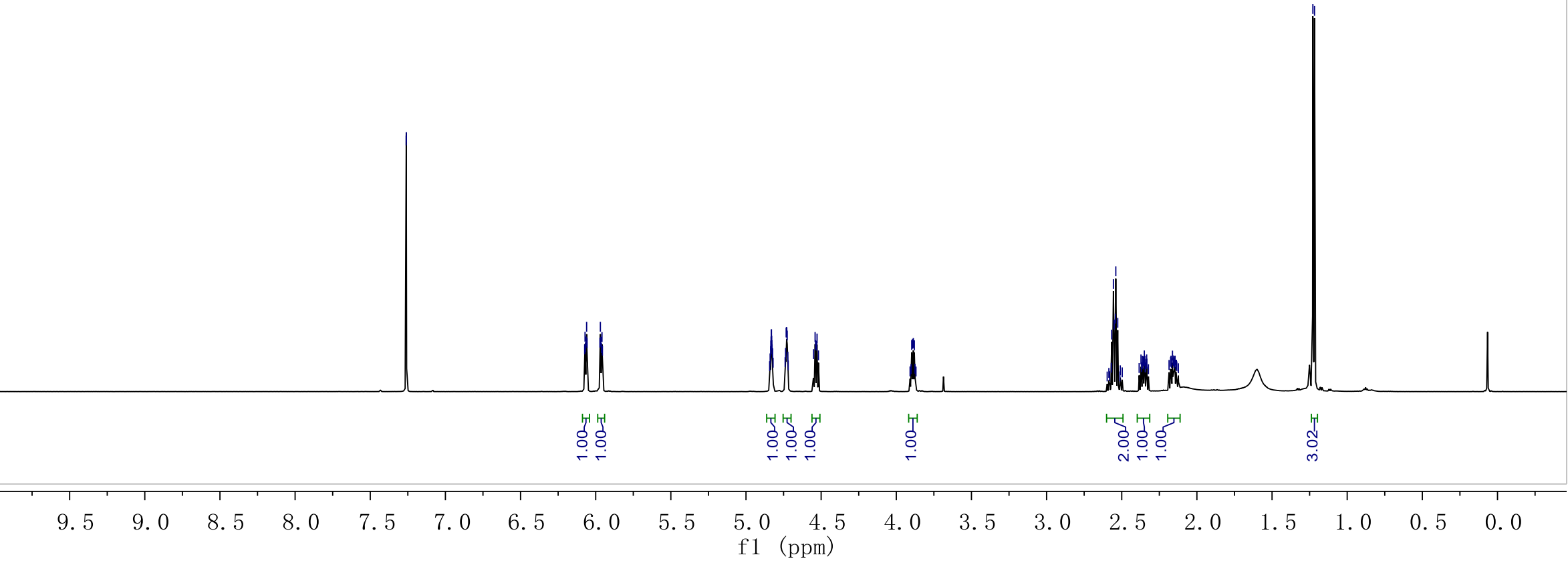



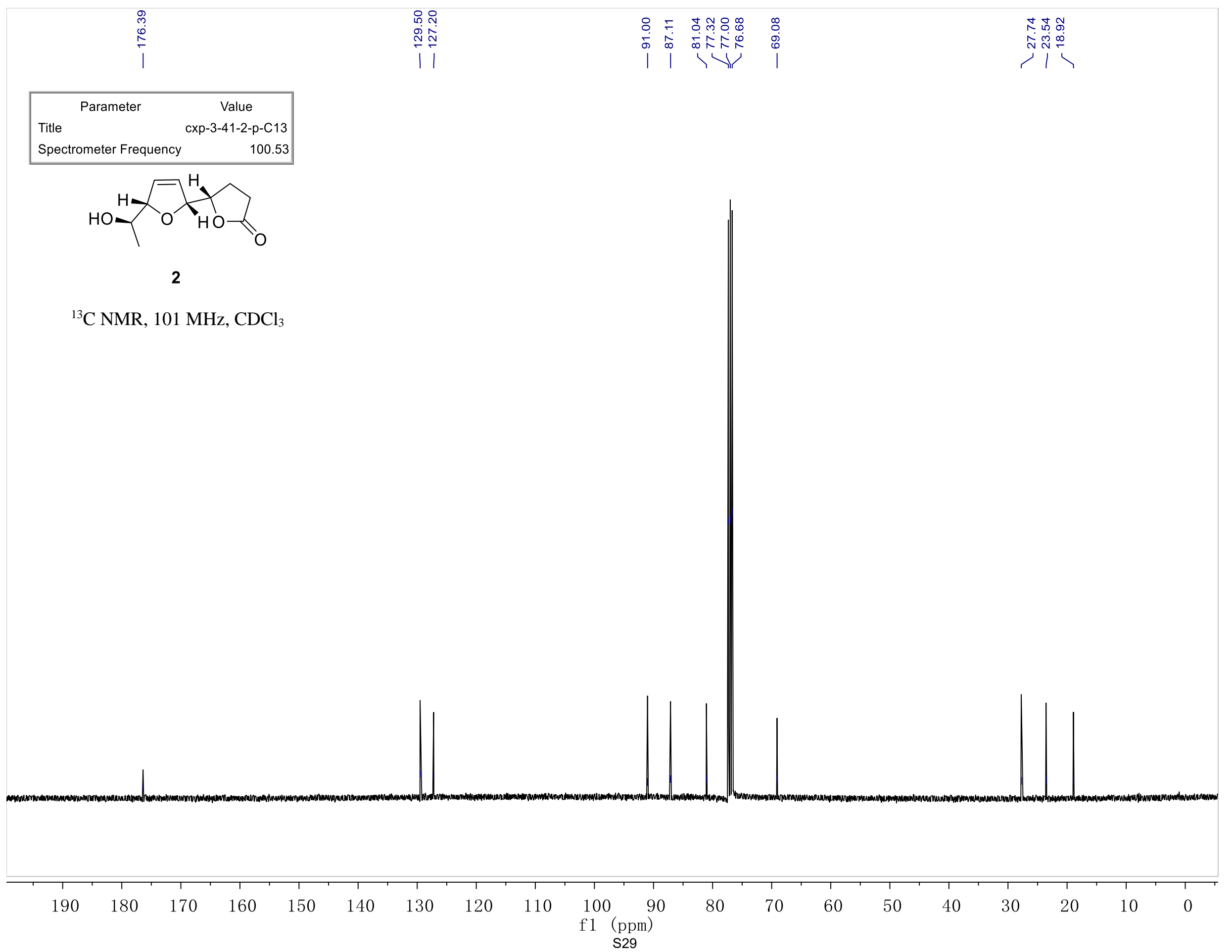


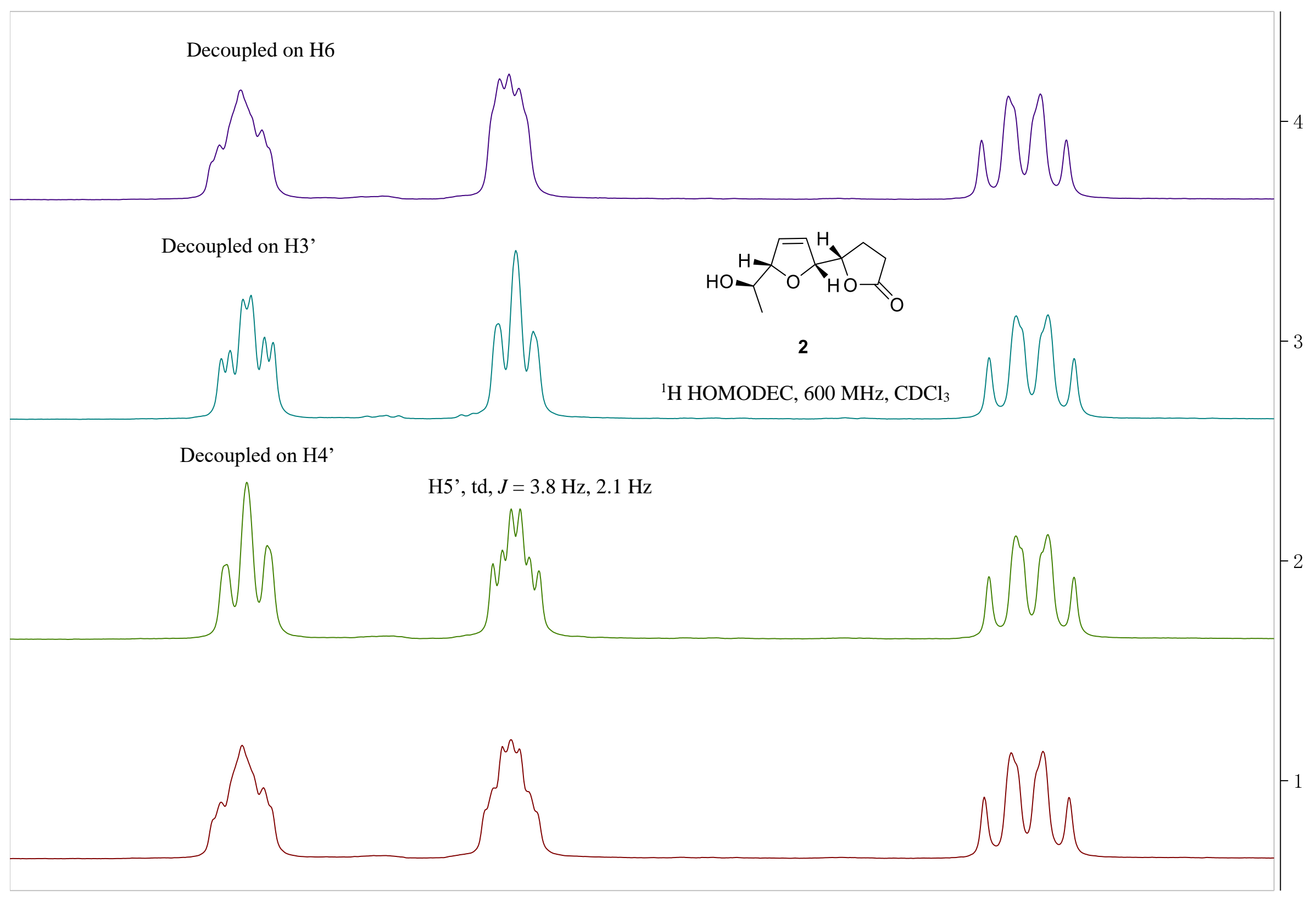

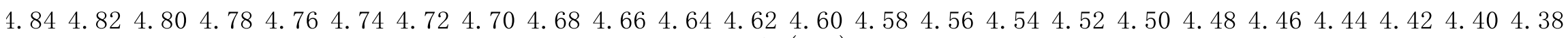
f1 (ppm) $)_{30}$ 
<smiles>C[C@H](O)[C@H]1C=C[C@H]([C@H]2CCC(=O)O2)O1</smiles>

2

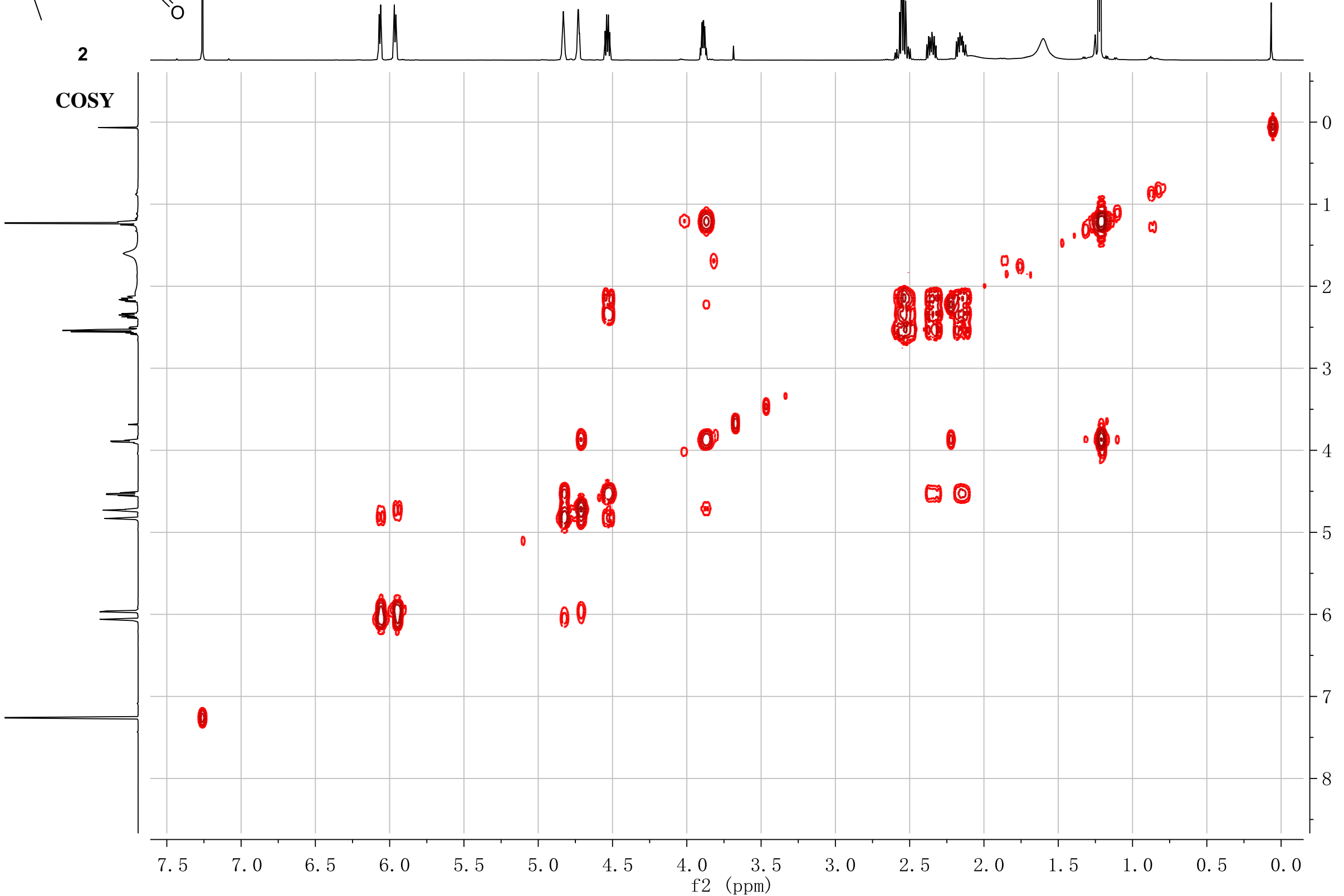

7. 5

7. 0

6. 5

6.0

5. 5

5. 0

f2 (ppm)

2.5

2. 0

1. 5

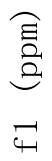

S31 


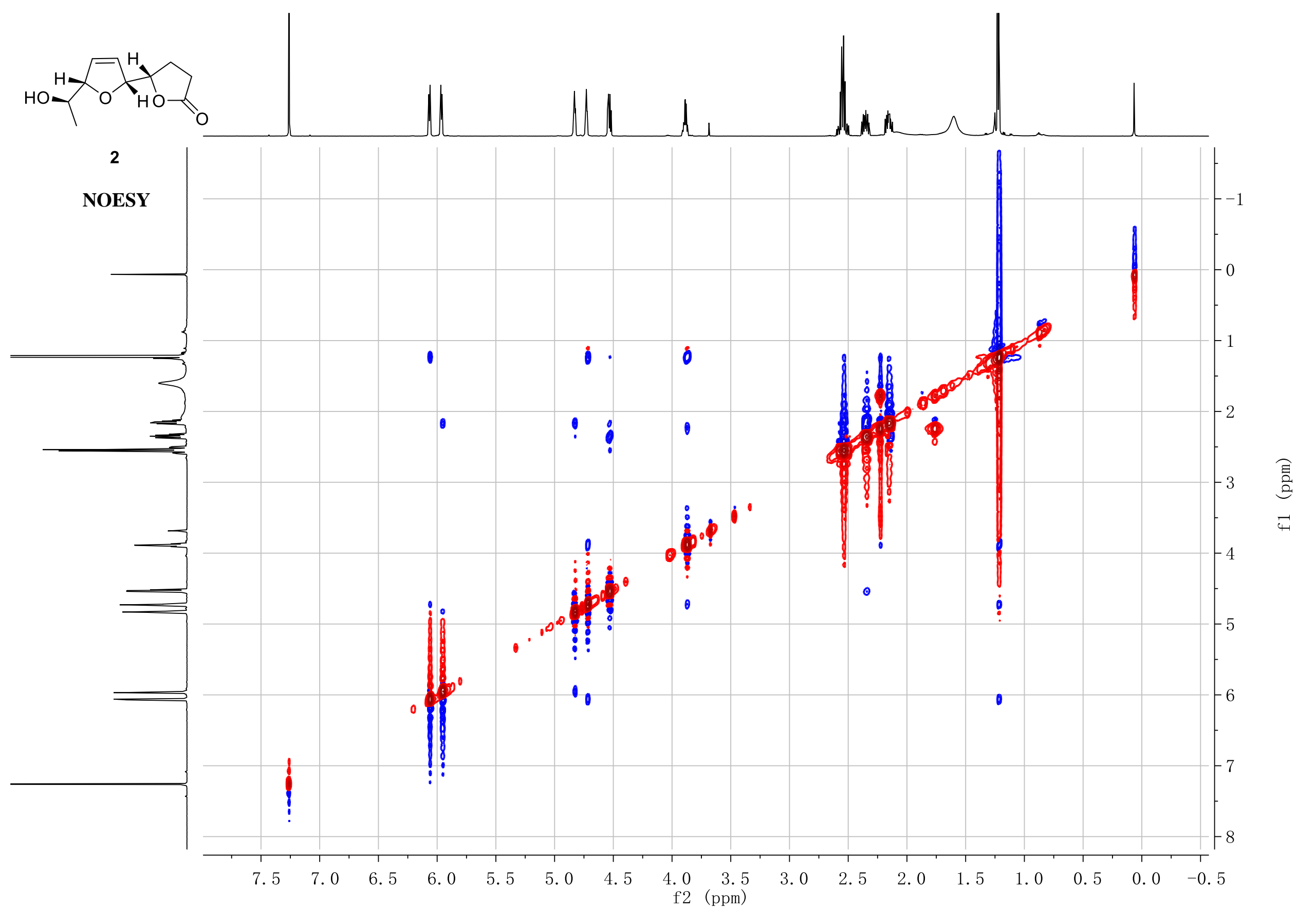




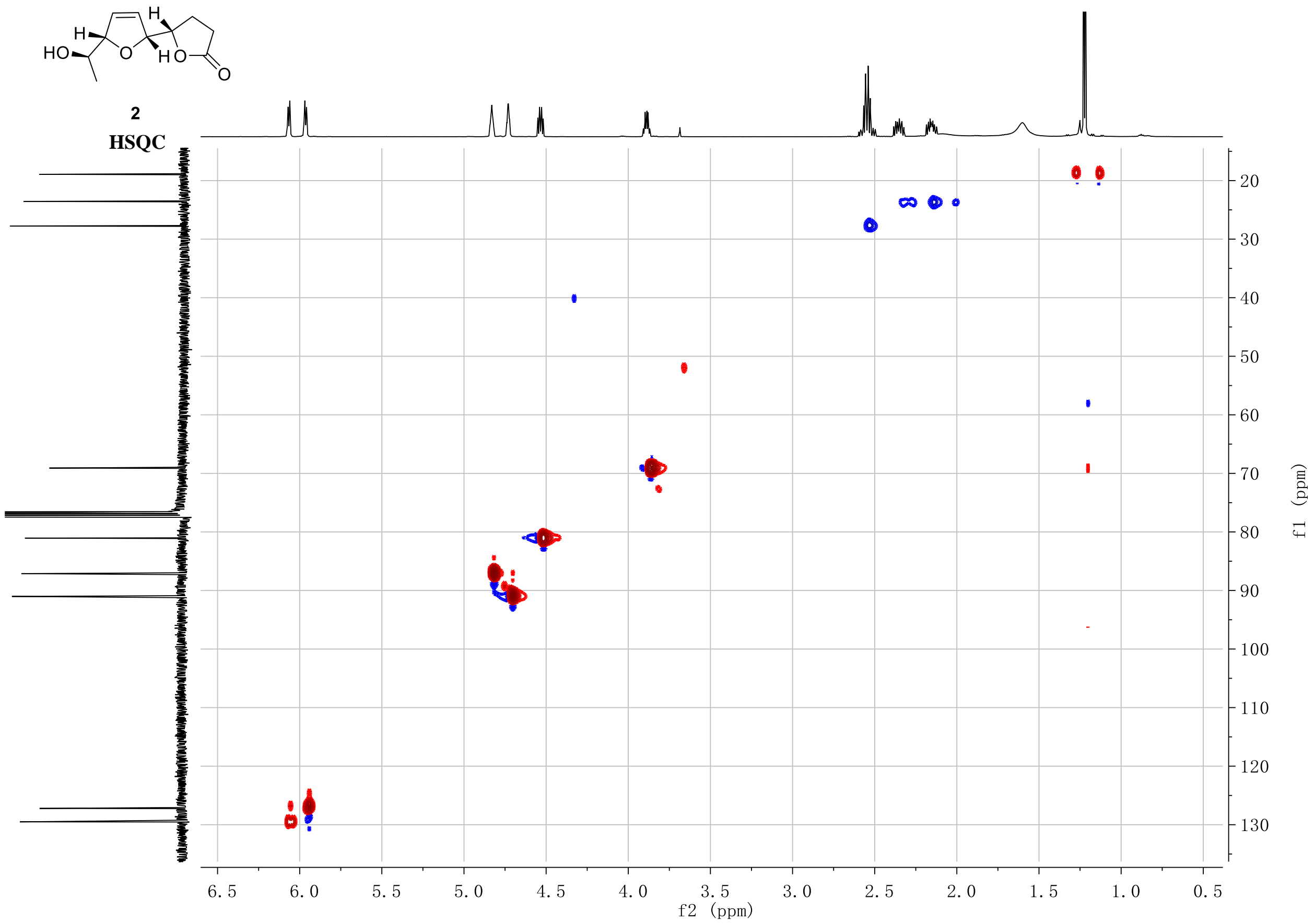

S33 


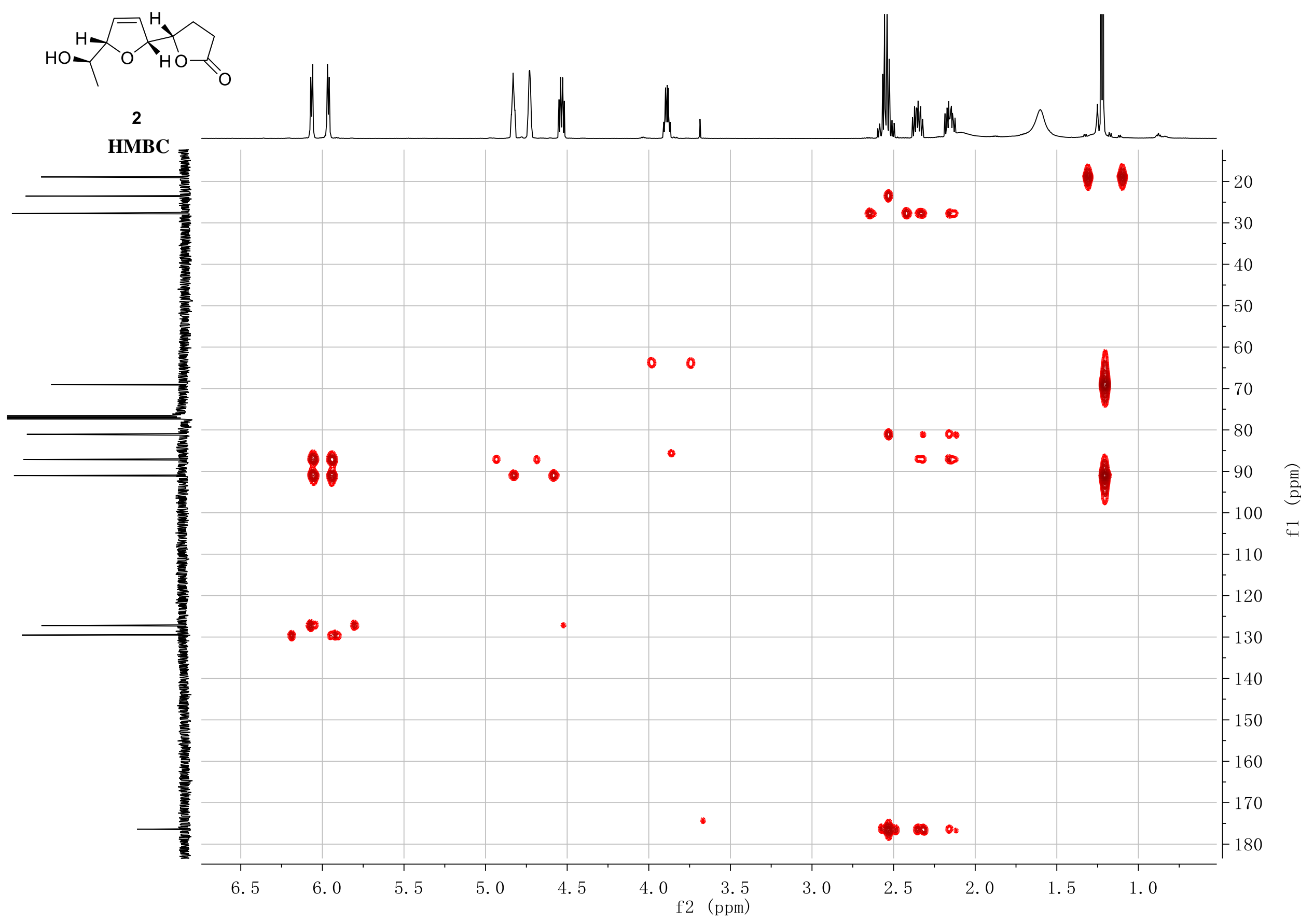



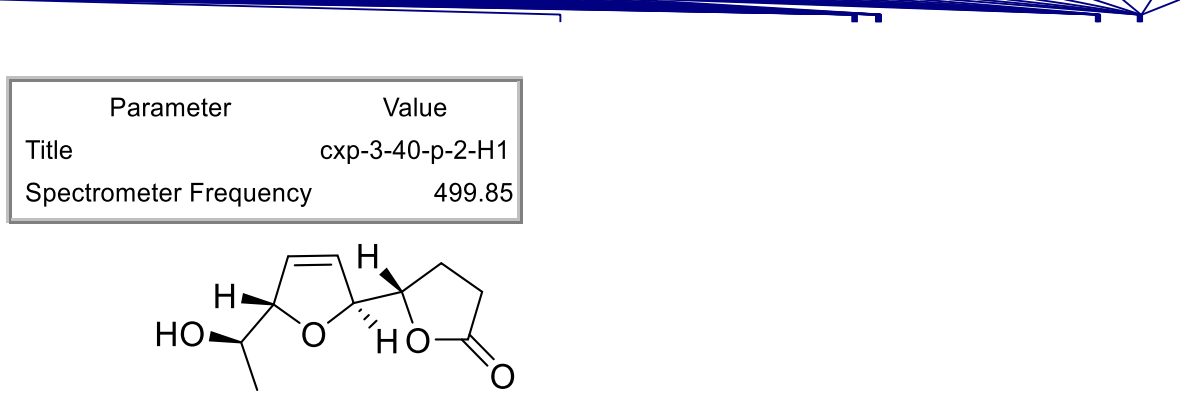

19

${ }^{1} \mathrm{H} \mathrm{NMR}, 600 \mathrm{MHz}, \mathrm{CDCl}_{3}$

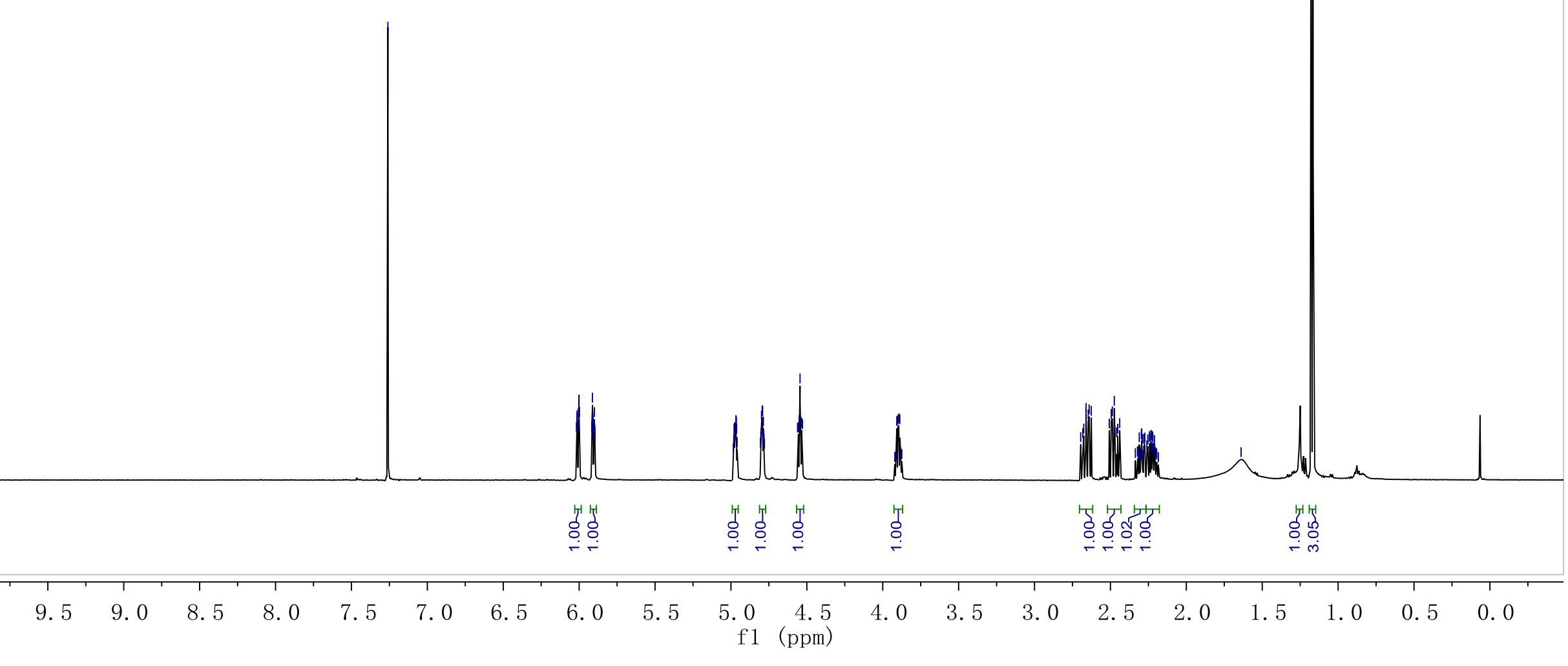



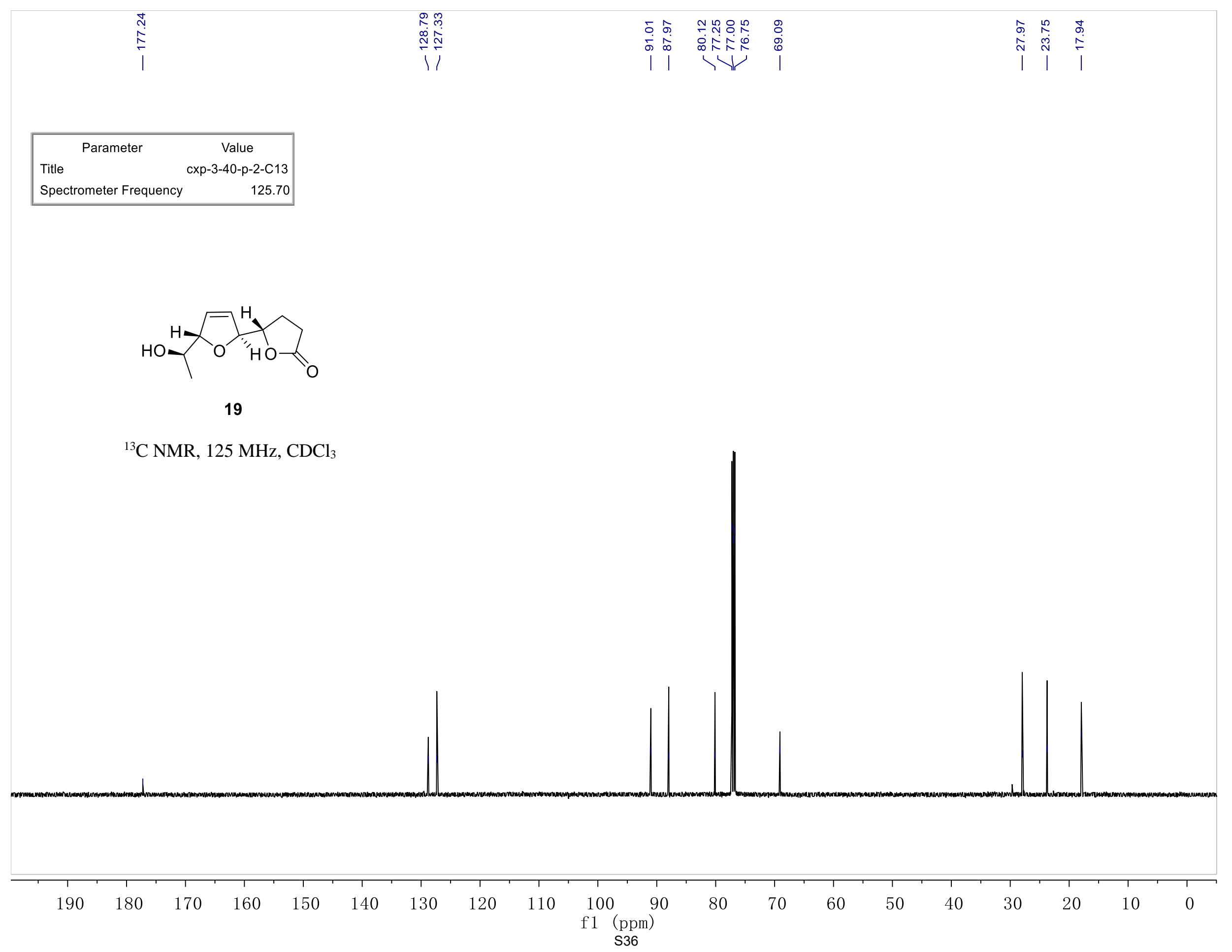


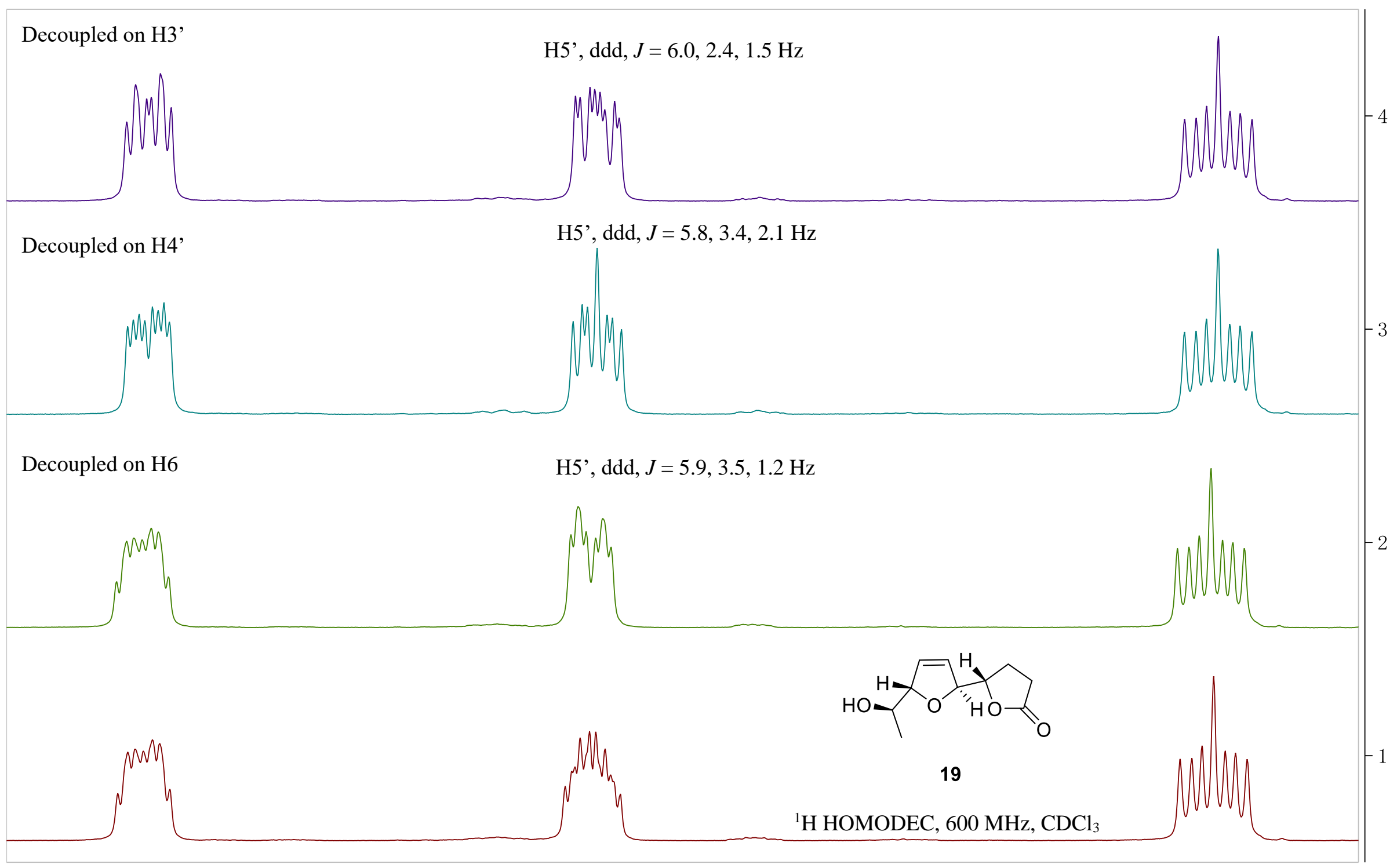

.025 .004 .984 .964 .944 .924 .904 .884 .864 .844 .824 .804 .784 .764 .744 .724 .704 .684 .664 .64 4.62 4. 604.584 .564 .544 .524 .50 f1 (ppm) 


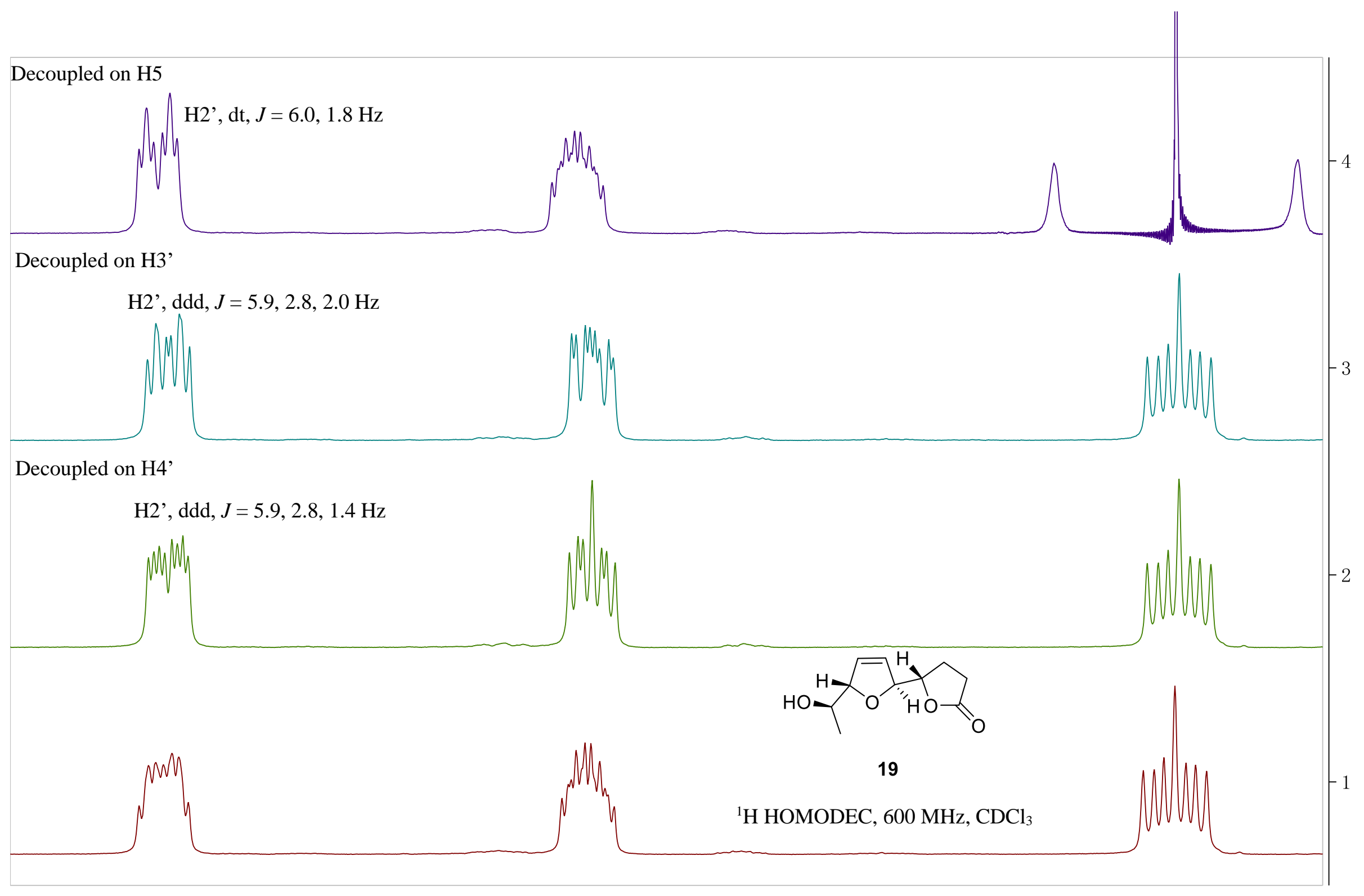

5. 00

4. 95

4. 90

4. 85

4. 80

4.75
(ppm)

4. 70

4. 65

4. 60

4. 55

4. 50 


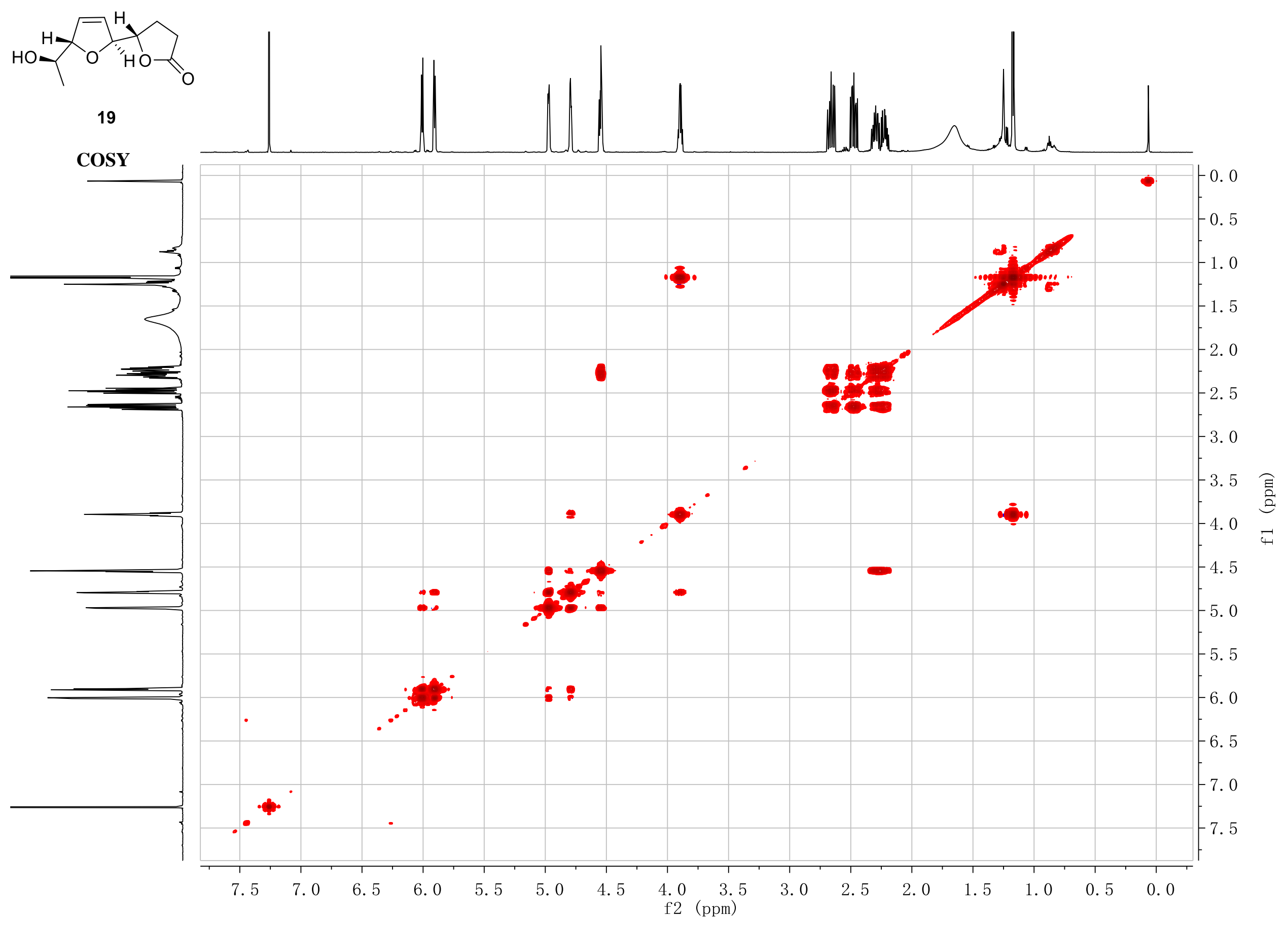




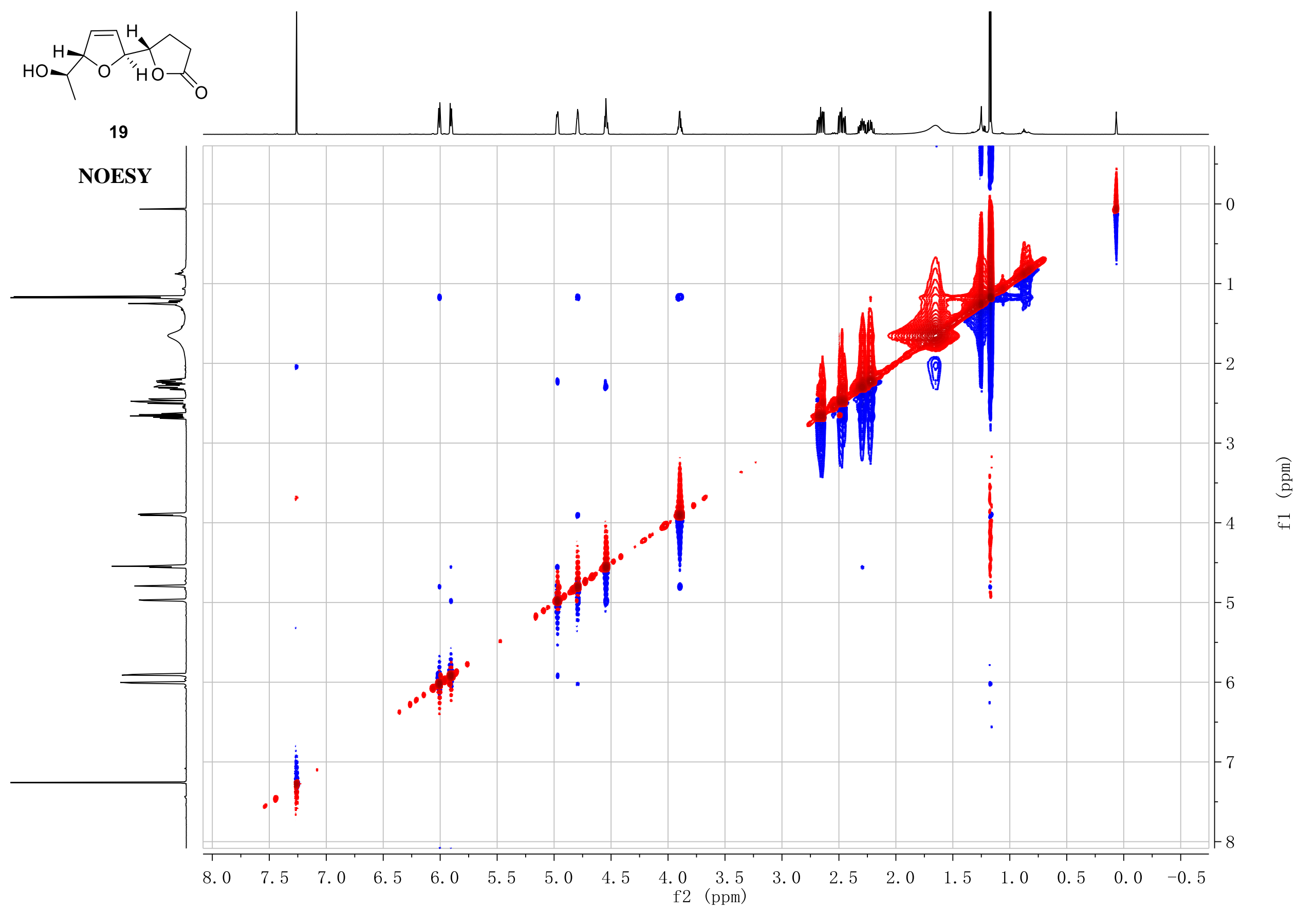




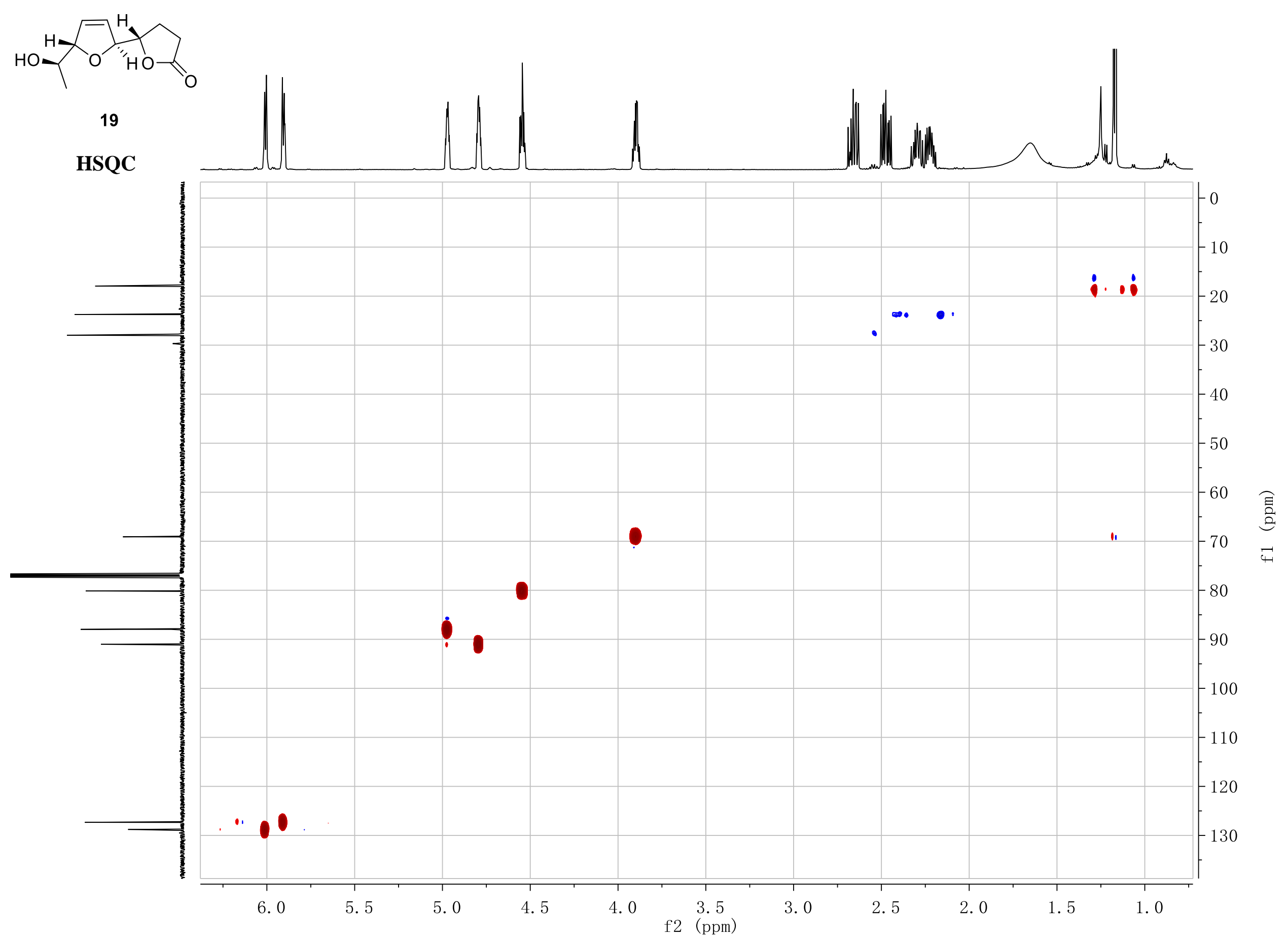




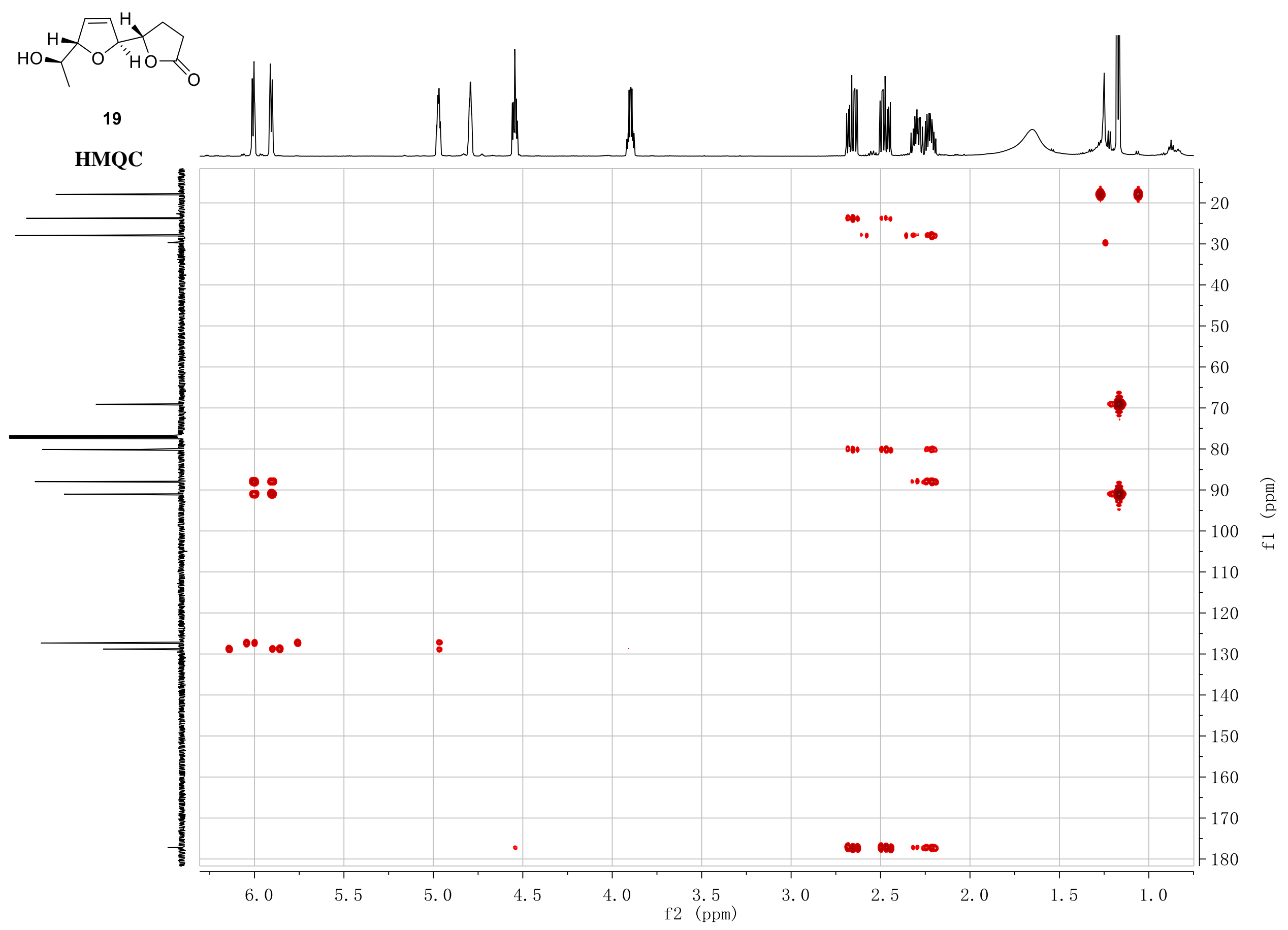




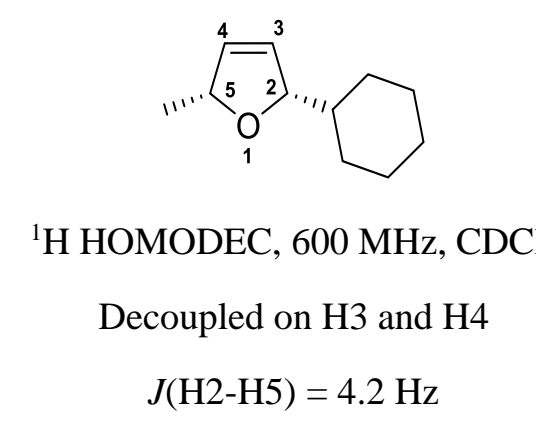

${ }^{1} \mathrm{H}$ HOMODEC, $600 \mathrm{MHz}, \mathrm{CDCl}_{3}$

Decoupled on $\mathrm{H} 3$ and $\mathrm{H} 4$

$J(\mathrm{H} 2-\mathrm{H} 5)=4.2 \mathrm{~Hz}$

H5, dd, $J=5.4,4.2 \mathrm{~Hz}$

$\mathrm{H} 2, \mathrm{qd}, J=6.4,4.2 \mathrm{~Hz}$

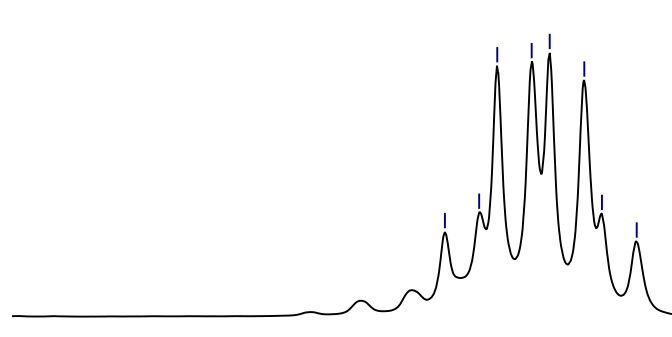

1

\begin{tabular}{|c|c|c|c|c|c|c|c|c|c|}
\hline 4.95 & 4. 90 & 4.85 & 4. 80 & 4. 75 & $1 \underset{\mathrm{Spm}}{\substack{\mathrm{ppm} \\
\mathrm{S} 43}}$ & 4.65 & 4.60 & 4. 55 & 4. 50 \\
\hline
\end{tabular}




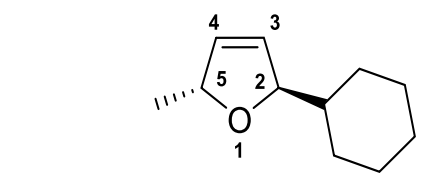

${ }^{1} \mathrm{H}$ HOMODEC, $600 \mathrm{MHz}, \mathrm{CDCl}_{3}$

Decoupled on $\mathrm{H} 3$ and $\mathrm{H} 4$

$J(\mathrm{H} 2-\mathrm{H} 5)=5.5 \mathrm{~Hz}$

$\mathrm{H} 5, \mathrm{t}, J=5.5 \mathrm{~Hz}$

$\mathrm{H} 2, \mathrm{~m}$

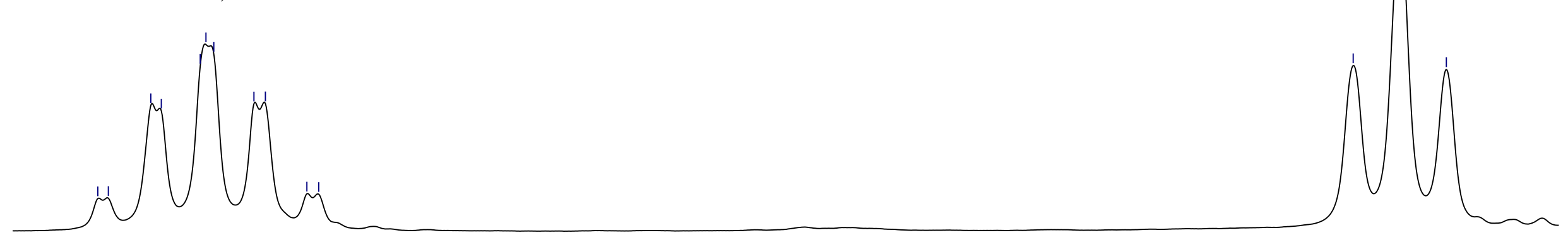

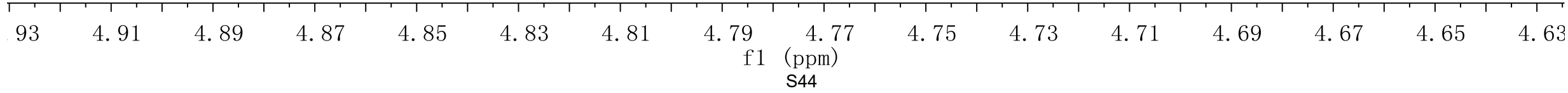

